Bruno Kahn $\cdot$ R. Sujatha

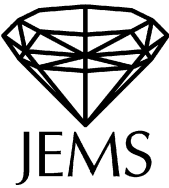

\title{
Motivic cohomology and unramified cohomology of quadrics
}

Received August 18, 1999 / final version received December 10, 1999

Published online April 19, 2000 - (c) Springer-Verlag \& EMS 2000

\begin{abstract}
This is the last of a series of three papers where we compute the unramified cohomology of quadrics in degree up to 4 . Complete results were obtained in the two previous papers for quadrics of dimension $\leq 4$ and $\geq 11$. Here we deal with the remaining dimensions between 5 and 10 . We also prove that the unramified cohomology of Pfister quadrics with divisible coefficients always comes from the ground field, and that the same holds for their unramified Witt rings. We apply these results to real quadrics. For most of the paper we have to assume that the ground field has characteristic 0 , because we use Voevodsky's motivic cohomology.
\end{abstract}

\section{Introduction}

This paper is the final part of our project of computation of the low-degree unramified cohomology of quadrics. Unramified cohomology groups are stable birational invariants of smooth projective varieties. They were originally introduced in relation with the so-called Noether problem (see [5], [4] for more details). These are subtle invariants, related to other interesting ones like Chow groups; they have been completely understood only for very few varieties. For varieties over a field $F$ stably birational to $\mathbf{P}^{n}$, they are determined by the Galois cohomology groups of $F$. After varieties birational to $\mathbf{P}^{n}$, the next simplest case is that of anisotropic quadrics. Yet another motivation for studying these groups is the conjecture in [15], which predicts that low rank unramified quadratic forms over function fields of quadrics over a field $F$ (of characteristic different from 2), are actually defined over the ground field $F$.

Throughout this paper, we assume that the ground field $F$ is of characteristic different from 2. In the later parts of the paper, we need $F$ to be of characteristic zero as we use Voevodsky's motivic cohomology [32].

B. Kahn: Institut de Mathématiques de Jussieu, Université Paris 7, Case 7012, 75251 Paris Cedex 05, France, e-mail: kahn@math.jussieu.fr

R. Sujatha: School of Mathematics, Tata Institute of Fundamental Research, Homi Bhabha Road, Bombay 400005, India, e-mail: sujatha@math.tifr.res.in

Mathematics Subject Classification (1991): 14F20, 14F42, 19D99 
Let $X$ be a smooth projective quadric defined over $F$ and $F(X)$ be its function field. For $n \geq 0$, recall that the $n$-th unramified cohomology group of the extension $F(X) / F$ with coefficients in $\mathbf{Q} / \mathbf{Z}(n-1)$, denoted $H_{\mathrm{nr}}^{n}(F(X) / F, \mathbf{Q} / \mathbf{Z}(n-1))$, is defined by (see [4])

$$
\begin{aligned}
& H_{\mathrm{nr}}^{n}(F(X) / F, \mathbf{Q} / \mathbf{Z}(n-1))= \\
& \quad \operatorname{Ker}\left(H^{n}(F(X), \mathbf{Q} / \mathbf{Z}(n-1)) \rightarrow \bigoplus_{x \in X^{(1)}} H^{n-1}(F(x), \mathbf{Q} / \mathbf{Z}(n-2))\right)
\end{aligned}
$$

where the cohomology groups are Galois cohomology groups, $F(x)$ is the residue field at a codimension one point $x \in X^{(1)}$ and the map comes from the residue homomorphisms; for $n=0$, the map is trivial. The natural restriction map in fact induces a map

$$
H^{n}(F, \mathbf{Q} / \mathbf{Z}(n-1)) \rightarrow H_{\mathrm{nr}}^{n}(F(X) / F, \mathbf{Q} / \mathbf{Z}(n-1))
$$

which we denote by $\eta_{X}^{n}$ as in our previous papers [17] and [18], or just by $\eta^{n}$, when there is no ambiguity on $X$. The principal results in these earlier papers are as follows. Firstly, we proved that Ker $\eta^{n}$ is generated by symbols for $n \leq 4$ and any $X$ (this is conjectured to hold without restriction on $n$ ). Secondly, we computed Coker $\eta^{3}$ for any $X$ and Coker $\eta^{4}$ (assuming char $F=0$ ) for any $X$ of dimension $\leq 4$. We showed that Coker $\eta^{3}$ is always 0 , except when $X$ is of dimension 4 and defined by an anisotropic Albert form, in which case this group has order 2. As for Coker $\eta^{4}$, we found that it is 0 for $\operatorname{dim} X \leq 3$. When $X$ is 4-dimensional (i.e. defined by a 6-dimensional form $q$ ), Coker $\eta^{4}$ is again 0 , except when $q$ is an Albert form or a "virtual Albert form". In these two cases, we found an intersting description of this group in terms of invariants attached to the orthogonal group of $q$. We also obtained some information on Coker $\eta^{4}$ when $\operatorname{dim} X>4$ : this group is canonically a subgroup of ${ }_{2} \mathrm{CH}^{3}(X)$, which is at most $\mathbf{Z} / 2$ and is 0 for $\operatorname{dim} X>10$ by results of Karpenko.

The contents of the present paper are as follows. First, we get more precise information on Coker $\eta^{4}$ when $\operatorname{dim} X>4$ in case char $F=0$. Second, we prove (still in characteristic 0 ) that Coker $\eta_{X}^{n}=0$ for all $n$ when $X$ is defined by a Pfister neighbour (Theorem 3 in Sect. 3). It follows that, for $X$ defined by a Pfister neighbour, the map

$$
W(F) \rightarrow W_{\mathrm{nr}}(F(X) / F)
$$

is surjective (Theorem 4 in the same section). The proof of this result makes full use of the techniques of Voevodsky in his proof of the Milnor conjecture [33]; however, we don't use the Milnor conjecture itself. Third and last, we give some computation of the $\mathcal{H}$-cohomology of real quadrics.

We now describe our results precisely. Let $X$ be a (smooth projective) quadric of dimension $>4$. By [17, Th. 6 (1)], there is an exact sequence 


$$
0 \rightarrow \operatorname{Coker} \eta^{4} \rightarrow C H^{3}(X)_{\text {tors }} \rightarrow H^{3}(F, 2) \oplus H^{5}(F, 3) .
$$

The proof of loc. cit., suitably adapted, in fact yields in characteristic zero an exact sequence

$$
0 \rightarrow \operatorname{Coker} \eta^{4} \rightarrow C H^{3}(X)_{\text {tors }} \stackrel{c l^{3}}{\rightarrow} H^{6}(X, \mathbf{Z}(3))
$$

( $c f$. also [16, diagram in 5.4]), which is the one we shall use here.

Theorem 1 gives a general result on quadrics $X$ of dimension $>6$, reducing the computation of Coker $\eta^{4}$ to that of ${ }_{2} \mathrm{CH}^{3}(\mathrm{X})$.

Theorem 1. Let $q$ be an anisotropic quadratic form of dimension $\geq 9$ over a field $F$ of characteristic 0 ; suppose that $q$ is not of the form $\pi \perp\langle a\rangle$ with $\pi$ similar to a 3-fold Pfister form. Let $X$ be the projective quadric defined by $q$. Then

a) The étale motivic cycle map

$$
c l^{3}: C H^{3}(X) \rightarrow H_{\text {ét }}^{6}(X, \mathbf{Z}(3))
$$

is trivial on $\mathrm{CH}^{3}(\mathrm{X})_{\mathrm{tors}}$.

b) There is an isomorphism

$$
\text { Coker } \eta^{4} \stackrel{\sim}{\rightarrow}{ }_{2} \mathrm{CH}^{3}(X)
$$

Corollary 1. Suppose in addition to the hypothesis of Theorem 1 that $F$ contains all 2-primary roots of unity and that $q$ is not a 4-fold Pfister neighbour. Then

$$
\text { Coker } \eta_{2}^{4} \simeq{ }_{2} \mathrm{CH}^{3}(X)
$$

where $\eta_{2}^{4}: H^{4}(F, \mathbf{Z} / 2) \rightarrow H_{\mathrm{nr}}^{4}(F(X) / F, \mathbf{Z} / 2)$ is the analogous map with $\mathbf{Z} / 2$-coefficients. Th. 1]

Indeed, in this case the map Coker $\eta_{2}^{4} \rightarrow$ Coker $\eta^{4}$ is an isomorphism [18,

Theorem 2 collects some cases in which we can actually determine Coker $\eta^{4}$.

Theorem 2. Let $q$ be a quadratic form of dimension $\geq 7$ over a field $F$ of characteristic 0 and $d_{ \pm} q$ denote its signed discriminant:

(i) If $\operatorname{dim} q=7$ and ind $c(q) \leq 2$, then Coker $\eta^{4}=0$.

(ii) If $\operatorname{dim} q=8$, then Coker $\eta^{4}=0$ in the following cases:

- $d_{ \pm} q=1$.

- $d=d_{ \pm} q \neq 1$ and $q_{F(\sqrt{d})}$ is similar to a Pfister form (which is automatically nonzero). 
(iii) If $\operatorname{dim} q=9$, then Coker $\eta^{4}=0$ in the following cases:

- $q$ represents its discriminant.

- $q$ contains an Albert form and ind $c(q) \neq 2$.

(iv) If $\operatorname{dim} q=10$, then Coker $\eta^{4}=0$ in the following cases:

- $d=d_{ \pm} q \neq 1, q_{F(\sqrt{d})}$ is isotropic but $\left(q_{F(\sqrt{d})}\right)_{\text {an }}$ is not similar to a nonzero Pfister form.

- $q$ contains an Albert form, $d_{ \pm} q=1$ and ind $c(q) \neq 2$.

(v) If $\operatorname{dim} q=11$, then Coker $\eta^{4}=0$ in the following cases:

- $q \simeq \tau \perp \psi$, with $\operatorname{dim} \tau=8, \tau \in I^{2} F \backslash I^{3} F, \operatorname{dim} \psi=3$ and $\tau_{F(\psi)}$ is not similar to an anisotropic Pfister form.

- $q$ contains an Albert form and $q \perp\left\langle-d_{ \pm} q\right\rangle \notin I^{3} F$.

(vi) If $\operatorname{dim} q=12$, then Coker $\eta^{4}=0$ in the following cases:

- $q \simeq \tau \perp \psi$, with $\operatorname{dim} \tau=8, \tau \in I^{2} F \backslash I^{3} F, \operatorname{dim} \psi=4$ and $\tau_{F(\psi)}$ is not similar to an anisotropic Pfister form.

- $q$ contains an Albert form and $q \notin I^{3} F$.

(vii) If $\operatorname{dim} q>12$, then Coker $\eta^{4}=0$.

Moreover, if $q$ is a generic quadratic form of dimension 12 such that $q \in$ $I^{3} F$, then Coker $\eta^{4} \neq 0$.

Theorems 1 and 2 represent by no means a complete computation of Coker $\eta^{4}$ for quadrics of dimension $>4$, and we felt happy to leave it to other mathematicians to take up our work from where we had left it. While we were completing this paper, we learnt that Oleg Izhboldin had done just this: in a remarkable piece of work [12], he supersedes Theorem 2 (iii)-(vii) by actually computing Coker $\eta_{X}^{4}$ for any quadric $X$ of dimension $>6$. The significance of this fact is that he uses the vanishing of this group when $X$ is defined by suitable types of 9-dimensional quadratic forms to construct the first known example of a field of $u$-invariant 9 .

Finally, here are our precise results for Pfister quadrics:

Theorem 3. Let $\varphi$ be an $n$-fold Pfister form over a field $F$ of characteristic 0 , $\psi$ a neighbour of $\varphi$ and $X$ the associated projective quadric. Then, for any $m \geq 0$, the map

$$
\eta^{m+1}: H^{m+1}(F, \mathbf{Q} / \mathbf{Z}(m)) \rightarrow H_{\mathrm{nr}}^{m+1}(F(X) / F, \mathbf{Q} / \mathbf{Z}(m))
$$

is surjective.

After proving this theorem, we learnt from A. Vishik that a key step of its proof, established independently, will also appear in the forthcoming paper [27].

Theorem 4. Let $\varphi, X$ be as in Theorem 3. Then, for any $n \geq 0$, the map

$$
I^{n} F \rightarrow I_{\mathrm{nr}}^{n}(F(X) / F)
$$

is surjective. 
Theorem 4 is all the more striking as, in general, the extension $F(X) / F$ is not excellent [11]. We think that this theorem should have an elementary proof, i.e. not using the Milnor conjecture or any Galois cohomology.

We can use these results to compute the cohomology groups $H^{p}\left(Q_{d}\right.$, $\left.\mathcal{H}^{q}(\mathbf{Z} / 2)\right)$ for real anisotropic quadrics $Q_{d}(c f . \S 4)$. For any smooth variety $X$, the cohomology groups $H^{p}\left(X, \mathcal{H}^{q}(\mathbf{Z} / 2)\right)$ occur as the $E_{2}^{p, q}$ terms of the Bloch-Ogus spectral sequence for mod 2 étale cohomology. These groups are known to be finite for real varieties whenever $q \geq d$, where $d=\operatorname{dim} X$ (see [30], for example). However, for $p+q<d$, even their finiteness is unknown in general. In [17], these groups were computed for all real quadrics when $p+q=4$. Here we compute these groups for $p+q=5$ and prove the injectivity of the cycle map $c l^{3}: \mathrm{CH}^{3}\left(Q_{d}\right) / 2 \rightarrow H_{e t}^{6}\left(Q_{d}, \mathbf{Z} / 2\right)$ (cf. Theorem 4.6).

\section{Torsion in $\mathrm{CH}^{3}$ of quadrics}

We collect here a few results on $C H^{3}$ of quadrics that are used in the next section. If $X$ is a smooth variety over $F$, we denote by $G^{*} K_{0}(X)$ the associated graded of the topological filtration on $K_{0}(X)$. For any $p$ there is a surjective map

$$
C H^{p}(X) \rightarrow G^{p} K_{0}(X)
$$

with kernel killed by $(p-1)$ !; in particular it is bijective for $p \leq 2$. Recall first

1.1. Lemma. [19, Cor. 4.5] If $X$ is a quadric, the above map is bijective for $p=3$.

Let $q$ be a quadratic form and $X_{q}$ the associated projective quadric. Recall from [21] that the elementary part of $C H^{p}\left(X_{q}\right)$ is by definition the subgroup generated by $h^{p}$, where $h$ is the class of a hyperplane section. The quotient $N E^{p}\left(X_{q}\right)=C H^{p}\left(X_{q}\right) /<h^{p}>$ is the nonelementary part of $\mathrm{CH}^{p}\left(X_{q}\right)$. The group $\mathrm{CH}^{p}\left(X_{q}\right)$ is elementary if $N E^{p}\left(X_{q}\right)=0$. The same definitions apply to the associated graded $G^{*} K_{0}\left(X_{q}\right)$ of the topological filtration on $K_{0}\left(X_{q}\right)$ (notation $N E K^{p}\left(X_{q}\right)$ for the non-elementary part). We have

1.2. Lemma. For $p \leq 3$, (2) induces an isomorphism

$$
N E^{p}(X) \stackrel{\sim}{\rightarrow} N E K^{p}(X)
$$

for any quadric $X$. 
Proof. This follows easily from Lemma 1.1 and the remarks preceding it.

If $\operatorname{dim} X_{q}>2 p$, or $\operatorname{dim} X_{q}=2 p, d_{ \pm} q \neq 1$, the natural map $C H^{p}\left(X_{q}\right)_{\text {tors }} \rightarrow$ $N E^{p}\left(X_{q}\right)$ is an isomorphism [19, (2.7)]. On the other hand, $N E^{p}\left(X_{q}\right) \neq 0$ if $p>\max \left(\operatorname{dim} X_{q} / 2, \operatorname{dim} X_{q}-i(q)\right)$, where $i(q)$ is the Witt index of $q$, or if $p=\operatorname{dim} X_{q} / 2, d_{ \pm} q=1$ (cf. [17, Prop. 1.1 (c) and (d)]). Moreover, if $q \simeq \varphi \perp \mathbf{H}$, then $N E^{p}\left(X_{q}\right) \simeq N E^{p-1}\left(X_{\varphi}\right)$ for $0<p<\operatorname{dim} X_{q}$, as follows from the isomorphism $C H^{p}\left(X_{q}\right) \simeq C H^{p-1}\left(X_{\varphi}\right)[19,(2.2)]$.

Also recall that, for a quadratic form $q, C H^{1}\left(X_{q}\right)$ is torsion-free [19, Prop. 2.4] and $C H^{2}\left(X_{q}\right)_{\text {tors }} \neq 0$ if and only if $q$ is a neighbour of an anisotropic 3-fold Pfister form, in which case $C H^{2}\left(X_{q}\right)_{\text {tors }} \simeq \mathbf{Z} / 2$ [19, Th. 6.1]. These facts and the previous ones will be used in the sequel without further mention.

The following complicated definition will be justified by Lemma 1.5, Proposition 1.6, Corollary 1.7 and Theorem 1.8.

1.3. Definition. Let $p \geq 0$.

a) A quadratic form $\varphi$ is $C H^{p}$-elementary (resp. $G^{p} K$-elementary) if $C H^{i}\left(X_{\varphi}\right)$ (resp. $G^{i} K_{0}\left(X_{\varphi}\right)$ ) is elementary for $i \leq p$.

b) The form $\varphi$ is stably $\mathrm{CH}^{p}$-elementary in codimension $i$ (resp. stably $G^{p} K$-elementary in codimension $i$ ) if it has the following property: for any extension $K / F$ and any quadratic form $q$ over $K$ containing $\varphi_{K}$, with $\operatorname{dim} q=\operatorname{dim} \varphi+i, q$ is $C H^{p}$-elementary (resp. $G^{p} K$-elementary).

c) It is stably $C H^{p}$-elementary (resp. stably $G^{p} K$-elementary) if it is stably $\mathrm{CH}^{p}$-elementary (resp. stably $G^{p} K$-elementary) in codimension $i$ for any $i \geq 0$.

d) It is stably $\mathrm{CH}^{p}$-elementary in codimension $>0$ (resp. stably $G^{p} \mathrm{~K}$-elementary in codimension $>0$ ) if it is stably $\mathrm{CH}^{p}$-elementary (resp. stably $G^{p} K$-elementary) in codimension $i$ for any $i>0$.

Examples.

1a) A quadratic form $\varphi$ of dimension $\geq 3$ is $C H^{1}$-elementary if and only if one of the following holds:

$-\operatorname{dim} \varphi>4$

$-\operatorname{dim} \varphi=4$ and $d_{ \pm} \varphi \neq 1$

- $\operatorname{dim} \varphi=3$ and $\varphi$ is anisotropic.

This follows easily from the remarks before Definition 1.3.

1b) A quadratic form $\varphi$ of dimension $\geq 3$ is stably $C H^{1}$-elementary if and only if $\operatorname{dim} \varphi>4$. Sufficiency is clear by the remarks before Definition 1.3. For necessity, note that if $\operatorname{dim} \varphi=4$ and $d_{ \pm} \varphi=1$, then $\varphi$ is not elementary, and that this condition is satisfied after some quadratic extension. 
2a) A quadratic form $\varphi$ of dimension $\geq 3$ is $\mathrm{CH}^{2}$-elementary if and only if one of the following holds:

- $\operatorname{dim} \varphi>6$ and $\varphi$ is not a neighbour of an anisotropic 3-fold Pfister form

$-\operatorname{dim} \varphi=6, d_{ \pm} \varphi \neq 1$ and $\varphi$ is not a neighbour of an anisotropic 3-fold Pfister form

- $\operatorname{dim} \varphi=5$ and $\varphi$ is not a neighbour of an anisotropic 3-fold Pfister form

$-\operatorname{dim} \varphi=5$ and $i(\varphi)<2$

$-\operatorname{dim} \varphi=4$ and $d_{ \pm} \varphi \neq 1$

- $\operatorname{dim} \varphi=3$ and $\varphi$ is anisotropic.

As in Example 1, this follows easily from the remarks before Definition 1.3.

2b) A quadratic form $\varphi$ of dimension $\geq 3$ is stably $\mathrm{CH}^{2}$-elementary if and only if one of the following holds:

$-\operatorname{dim} \varphi>8$

- $\operatorname{dim} \varphi=8$ and $\varphi$ contains an Albert form (i.e. $\varphi_{F\left(\sqrt{d_{ \pm} \varphi}\right)}$ is isotropic)

- $\operatorname{dim} \varphi=7$ and $\varphi$ contains an Albert form (i.e. $\varphi \perp<-d_{ \pm} \varphi>$ is isotropic).

Sufficiency is clear by the remarks before Definition 1.3: the conditions imply that $\varphi$ is not a neighbour of a 3-fold Pfister form, and they are clearly invariant under base field extension. Although we don't really need necessity in the sequel, we prove it for the reader's pleasure. We first note that none of the conditions in 2a) is stable under base field extension if $\operatorname{dim} \varphi \leq 6$. For example, if $\operatorname{dim} \varphi=5, \varphi$ is anisotropic and not a Pfister neighbour, then $\varphi$ becomes an anisotropic Pfister neighbour over $F\left(X_{\varphi \perp\left\langle-d_{ \pm} \varphi\right\rangle}\right)$ (this is the only non obvious case). Also, any anisotropic 7-dimensional form which does not contain an Albert form is contained in an anisotropic 8-dimensional form of discriminant 1 . It is therefore sufficient to show that, if $\operatorname{dim} \varphi=8$ and $\varphi$ does not contain an Albert form, then $\varphi$ becomes similar to an anisotropic Pfister form over some extension of the base field. Using a quadratic extension, we can assume that $d_{ \pm} \varphi=1$. To make $\varphi$ similar to a Pfister form, we pass to the function field $K$ of the Severi-Brauer variety of $C(\varphi)$. There remains to see that $\varphi_{K}$ is anisotropic. If ind $C(\varphi) \leq 4$, this is [23, Th. 4], and if ind $C(\varphi)=8$, this is [9, Th. 9.1].

2c) A quadratic form $\varphi$ of dimension $\geq 3$ is stably $C H^{2}$-elementary in codimension 1 if one of the following holds:

$-\operatorname{dim} \varphi \geq 8$

- $\operatorname{dim} \varphi=7$ and $\varphi$ contains an Albert form

- $\operatorname{dim} \varphi=6$ and $\varphi$ is a (possibly isotropic) Albert form. 
The proof is easy. (Conversely, one easily sees that no form of dimension $\leq 5$ is stably $\mathrm{CH}^{2}$-elementary in codimension 1 and that the condition for 7-dimensional forms is necessary.) Note that, by Remark 1b), all these forms are also stably $C H^{1}$-elementary.

1.4. Lemma. a) Let $X$ be a smooth variety over $F$. Then, for any $p \geq 0$, the map $\mathrm{CH}^{p}(X) \rightarrow \mathrm{CH}^{p}\left(X_{F(t)}\right)$ is bijective.

b) If $X$ is a quadric, then $N E^{p}(X) \stackrel{\sim}{\rightarrow} N E^{p}\left(X_{F(t)}\right)$.

Proof. a) Surjectivity follows from the commutative diagram

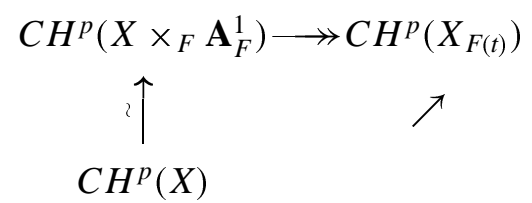

where the vertical map is bijective by homotopy invariance. Injectivity follows from a classical specialisation argument (using the existence of closed points of coprime degrees in any open subset of $\mathbf{A}_{F}^{1}$ ). b) follows immediately from a).

The following lemma and proposition slightly refine the substance of [21, Lemma 6.2].

1.5. Lemma. Let $\varphi$ be a quadratic form over $F, C H^{p-1}$-elementary, stably $\mathrm{CH}^{p-2}$-elementary in codimension 0 and stably $\mathrm{CH}^{p-1}$-elementary in codimension 1. Let $\psi=\left\langle a_{1}, a_{2}\right\rangle$, and suppose that $\varphi_{E}$ is $C H^{p-1}$-elementary, where $E=F\left(\sqrt{-a_{1} a_{2}}\right)$. Let $K=F(t)$ and $\tilde{\psi}=a_{1} t^{2}+a_{2} \in K^{*}$. Then there is a natural isomorphism

$$
N E^{p}\left(X_{\varphi \perp \psi}\right) \simeq N E^{p}\left(X_{\varphi_{K} \perp\langle\tilde{\psi}\rangle}\right) .
$$

Proof. We proceed exactly as in [21]. There is an exact sequence

$$
C H^{p-1}\left(X_{\varphi \perp\left\langle a_{1}\right\rangle}\right) \rightarrow C H^{p}\left(X_{\varphi \perp\left\langle a_{1}, a_{2}\right\rangle}\right) \rightarrow C H^{p}(U) \rightarrow 0
$$

where $U$ is the affine quadric $X_{\varphi \perp\left\langle a_{1}, a_{2}\right\rangle} \backslash X_{\varphi \perp\left\langle a_{1}\right\rangle}$. From it and the definition of $N E^{*}$, we deduce another exact sequence

$$
N E^{p-1}\left(X_{\varphi \perp\left\langle a_{1}\right\rangle}\right) \rightarrow N E^{p}\left(X_{\varphi \perp\left\langle a_{1}, a_{2}\right\rangle}\right) \rightarrow C H^{p}(U) \rightarrow 0 .
$$

On the other hand, projection on the second coordinate gives a morphism $U \rightarrow \mathbf{A}^{1}$, hence another exact sequence

$$
\coprod_{\alpha \in \mathbf{A}_{F}^{1}} C H^{p-1}\left(U_{\alpha}\right) \rightarrow C H^{p}(U) \rightarrow C H^{p}\left(U_{\eta}\right) \rightarrow 0
$$


where the left summation is over all closed points of $\mathbf{A}_{F}^{1}$, and $\eta$ is the generic point of $\mathbf{A}_{F}^{1}$.

For all $\alpha, U_{\alpha}$ is the affine quadric $X_{\varphi_{F(\alpha)} \perp\left\langle x_{\alpha}\right\rangle} \backslash X_{\varphi}$ over the residue field $F(\alpha)$, where $x_{\alpha}$ is the image of $a_{1} t^{2}+a_{2}$ in $F(\alpha)$. If $x_{\alpha} \neq 0$, the form $\varphi_{F(\alpha)} \perp\left\langle x_{\alpha}\right\rangle$ is nondegenerate. If $x_{\alpha}=0$ (let us denote the corresponding point $\alpha$ by $\left.\alpha_{0}\right)$, then $F\left(\alpha_{0}\right)=E$. By [21, Lemma 5.3], $C H^{p-1}\left(U_{\alpha_{0}}\right)$ gets identified with $\operatorname{Coker}\left(C^{p-2}\left(X_{\varphi_{E}}\right) \stackrel{. h}{\rightarrow} C H^{p-1}\left(X_{\varphi_{E}}\right)\right)$. Finally, from (3) and (4), we get a commutative diagram with exact row and columns

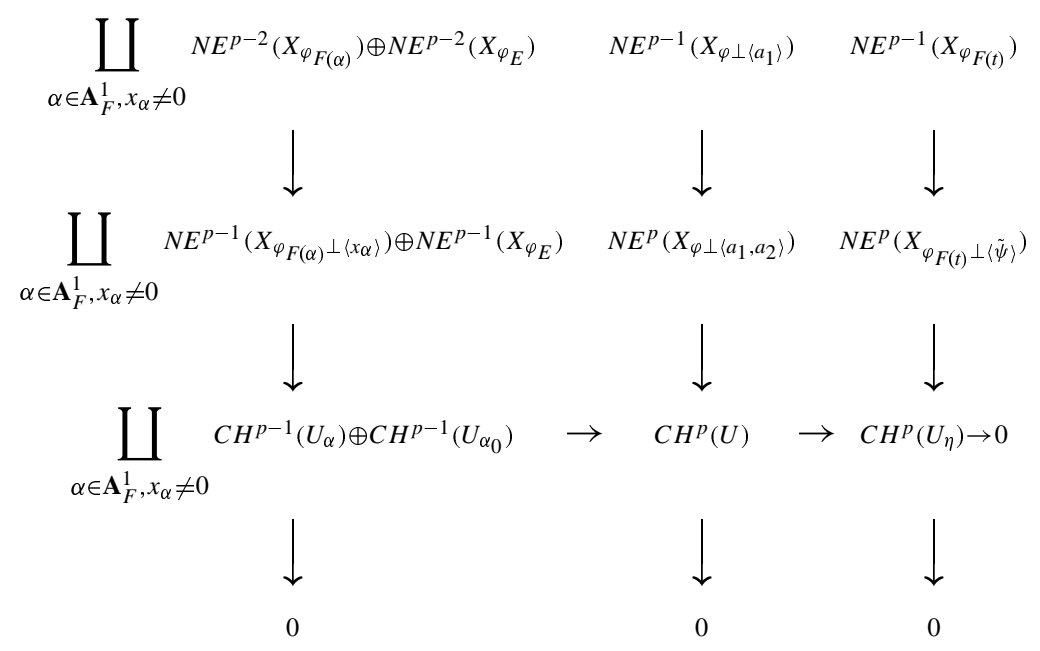

Let us now use the assumptions on $\varphi$. Since $\varphi$ is $C H^{p-1}$-elementary, Lemma 1.4 implies that $N E^{p-1}\left(X_{\varphi_{F(t)}}\right)=0$. Since $\varphi$ is stably $\mathrm{CH}^{p-2}$ elementary in codimension 0 , the top left term in the diagram is 0 too. Since $\varphi$ is stably $\mathrm{CH}^{p-1}$-elementary in codimension 1 , the top middle term is 0 , as well as the first summand of the middle left term. Finally, since $\varphi_{E}$ is $\mathrm{CH}^{p-1}$-elementary, the second summand of the middle left term is 0 too. Hence the bottom left term is 0 and the diagram simplifies as

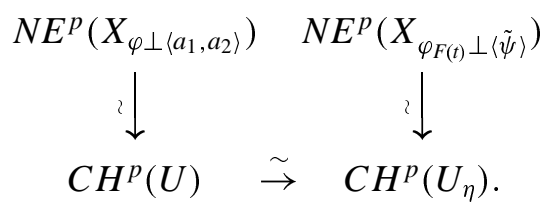

Hence Lemma 1.5.

1.6. Proposition. Let $\varphi$ be quadratic form over $F$, and let $r \geq 2$. Suppose that $\varphi$ is stably $\mathrm{CH}^{p-2}$-elementary, $\mathrm{CH}^{p-1}$-elementary and stably $\mathrm{CH}^{p-1}$ elementary in codimension $i$ for all $i \in] 0, r\left[\right.$. Let $\psi=\left\langle a_{1}, \ldots, a_{r}\right\rangle$ be another form, and suppose finally that $\varphi_{F\left(X_{\psi}\right)}$ is $\mathrm{CH}^{p-1}$-elementary. Let 
$K=F\left(t_{1}, \ldots, t_{r-1}\right)$ and $\tilde{\psi}=a_{1} t_{1}^{2}+\ldots a_{r-1} t_{r-1}^{2}+a_{r} \in K^{*}$. Then there is a natural isomorphism

$$
N E^{p}\left(X_{\varphi \perp \psi}\right) \simeq N E^{p}\left(X_{\varphi_{K} \perp\langle\tilde{\psi}\rangle}\right) .
$$

Proof. We proceed by induction on $r$, the case $r=2$ having been dealt with in Lemma 1.5. Let $\varphi^{\prime}=\varphi \perp\left\langle a_{1}\right\rangle$ and $\psi^{\prime}=\left\langle a_{2}, \ldots, a_{r}\right\rangle$, so that $\varphi \perp$ $\psi \simeq \varphi^{\prime} \perp \psi^{\prime}$. Then $\varphi^{\prime}$ verifies the first three assumptions of Proposition 1.6 (relatively to $r-1$ for the third one). Therefore

$$
N E^{p}\left(X_{\varphi \perp \psi}\right)=N E^{p}\left(X_{\varphi^{\prime} \perp \psi^{\prime}}\right) \simeq N E^{p}\left(X_{\varphi_{L}^{\prime} \perp\left\langle\tilde{\psi}^{\prime}\right\rangle}\right)
$$

with $L=F\left(t_{2}, \ldots, t_{r-1}\right)$ and $\tilde{\psi}^{\prime}=a_{2} t_{2}^{2}+\ldots a_{r-1} t_{r-1}^{2}+a_{r} \in L^{*}$. We have

$$
\varphi_{L}^{\prime} \perp\left\langle\tilde{\psi}^{\prime}\right\rangle \simeq \varphi_{L} \perp\left\langle a_{1}, \tilde{\psi}^{\prime}\right\rangle .
$$

The form $\varphi_{L}$ is $C H^{p-1}$-elementary by Lemma 1.4 , stably $\mathrm{CH}^{p-2}$ elementary in codimension 0 and stably $C H^{p-1}$-elementary in codimension 1. Moreover $\varphi_{E}$ is $C H^{p-1}$-elementary, where $E=L\left(\sqrt{-a_{1} \tilde{\psi}^{\prime}}\right)=$ $F\left(X_{\psi}\right)$. Therefore the triple $\left(L, \varphi_{L},\left\langle a_{1}, \tilde{\psi}^{\prime}\right\rangle\right)$ satisfies the conditions of Lemma 1.5. Hence we have

$$
N E^{p}\left(X_{\varphi_{L}^{\prime} \perp\left\langle\tilde{\psi}^{\prime}\right\rangle}\right) \simeq N E^{p}\left(X_{\varphi_{K} \perp\langle\tilde{\psi}\rangle}\right)
$$

and the proof is complete.

1.7. Corollary. A form stably $\mathrm{CH}^{p-1}$-elementaryand stably $\mathrm{CH}^{p}$-elementary in codimension 1 is stably $\mathrm{CH}^{p}$-elementary in codimension $>0$.

This corollary allows us to give a slightly stronger variant of Proposition 1.6:

1.8. Theorem. Let $\varphi$ be stably $\mathrm{CH}^{p-2}$-elementary, $\mathrm{CH}^{p-1}$-elementary and stably $\mathrm{CH}^{p-1}$-elementary in codimension 1 . Let $\psi=\left\langle a_{1}, \ldots, a_{r}\right\rangle$ be another form, and suppose that $\varphi_{F\left(X_{\psi}\right)}$ is $\mathrm{CH}^{p-1}$-elementary. Let $K=$ $F\left(t_{1}, \ldots, t_{r-1}\right)$ and $\tilde{\psi}=a_{1} t_{1}^{2}+\ldots a_{r-1} t_{r-1}^{2}+a_{r} \in K^{*}$. Then there is a natural isomorphism

$$
N E^{p}\left(X_{\varphi \perp \psi}\right) \simeq N E^{p}\left(X_{\varphi_{K} \perp\langle\tilde{\psi}\rangle}\right) .
$$

This applies notably with $p=3$ and $\varphi$ as in Example 2c) after Definition 1.3.

We now observe that the proof of [21, Cor. 4.9] in fact yields the following (cf. loc. cit., Lemma 7.2): 
1.9. Proposition. Let $\varphi$ be a quadratic form over $F$ and $E / F$ a finite extension such that the norm map

$$
N_{E / F}: K_{0}\left(C_{0}\left(\varphi_{E}\right)\right) \rightarrow K_{0}\left(C_{0}(\varphi)\right)
$$

is surjective (e.g. E may be any subfield of the division algebra derived from $C_{0}(\varphi)$ ). If, for some $p, \varphi_{E}$ is $G^{p-1} K$-elementary, then the map

$$
N_{E / F}: N E K^{i}\left(X_{\varphi_{E}}\right) \rightarrow N E K^{i}\left(X_{\varphi}\right)
$$

is surjective for $i \leq p$.

Proof. We sketch a proof for the reader's convenience. The assumption that $\varphi_{E}$ is $G^{p-1} K$-elementary implies that (cf. loc. cit., Lemma 4.4)

$$
N E K^{i}\left(X_{\varphi_{E}}\right)=\operatorname{Coker}\left(K_{0}\left(X_{\varphi_{E}}\right)^{i+1} \rightarrow K_{0}\left(X_{\varphi_{E}}\right) /<H>\right)
$$

for $i \leq p$, where $<H>$ denotes the subgroup generated by the elements $h^{i}$. We have a commutative diagram with surjective horizontal maps [21, Th. 4.2]

$$
\begin{aligned}
& K_{0}\left(C_{0}\left(\varphi_{E}\right)\right) \longrightarrow K_{0}\left(X_{\varphi_{E}}\right) /<H> \\
& N_{E / F} \downarrow \quad N_{E / F} \downarrow \\
& K_{0}\left(C_{0}(\varphi)\right) \longrightarrow K_{0}\left(X_{\varphi}\right) /<H>\text {. }
\end{aligned}
$$

Thus the hypothesis that the left vertical map is surjective implies the surjectivity of the right vertical arrow. The proposition now follows on using this surjectivity (with $i \leq p$ ) in the following natural diagram with exact rows

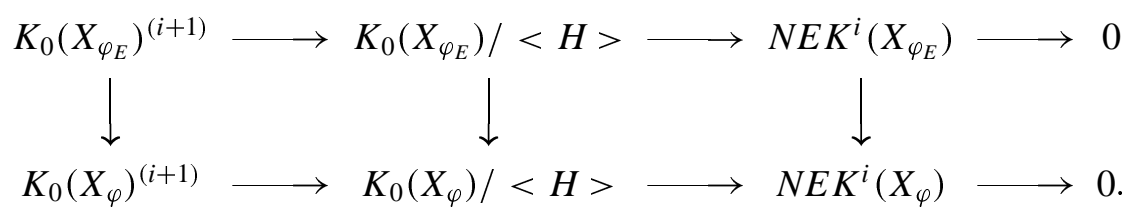

Similarly, the proof of $[21$, Cor. 4.5$]$ in fact gives

1.10. Proposition. Let $\psi$ be an odd-dimensional quadratic form over $F$ and set $\varphi=\psi \perp\left\langle-d_{ \pm} \psi\right\rangle$. If, for some $p, \varphi$ is $G^{p-1} K$-elementary, then the pull-back map

$$
\operatorname{NEK}^{i}\left(X_{\varphi}\right) \rightarrow \operatorname{NEK} K^{i}\left(X_{\psi}\right)
$$

is surjective for $i \leq p$. 
1.11. Corollary. Let $\tau \in I^{2} F$ such that $\tau$ is $G^{p-2} K$-elementaryfor some $p$. Let $d \in F^{*}$. Then the push-forward map

$$
N E K^{i-1}\left(X_{\tau}\right) \rightarrow N E K^{i}\left(X_{\tau \perp\langle d\rangle}\right)
$$

is surjective for $i \leq p$.

Proof. Let $\varphi=\psi \perp\langle-d\rangle \simeq \tau \perp \mathbf{H}$. For $i \leq p-1$, we have $G^{i} K_{0}\left(X_{\varphi}\right)$ $\simeq G^{i-1} K_{0}\left(X_{\tau}\right)$, hence $\varphi$ is $G^{p-1} K$-elementary. It remains to see that the composition

$$
G^{i-1} K_{0}\left(X_{\tau}\right) \stackrel{\sim}{\rightarrow} G^{i} K_{0}\left(X_{\varphi}\right) \rightarrow G^{i} K_{0}\left(X_{\psi}\right)
$$

coincides with the push-forward map, which is clear from the construction of the first isomorphism.

Also, the proof of [21, Cor. 4.7] in fact gives

1.12. Proposition. Let $\tau$ be an even-dimensional quadratic form of nontrivial discriminant $d$. Suppose that $\tau$ contains a 1-codimensional subform $\varphi$ such that the quadratic extension $E=F(\sqrt{d})$ preserves ind $C_{0}(\varphi)$. If, for some $p, \varphi$ is $G^{p-2} K$-elementary, then the push-forward map

$$
N E K^{i-1}\left(X_{\varphi}\right) \rightarrow N E K^{i}\left(X_{\tau}\right)
$$

is surjective for $i \leq p$.

Finally, the following proposition captures the substance of the argument in the proof of [21, Prop. 6.3].

1.13. Proposition. Let $\varphi$ be an odd-dimensional form and $\psi$ another form. Write $C_{0}(\varphi)=M_{n}(D)$, where $D$ is a division algebra. Assume that $(\varphi, \psi)$ verifies the conditions of Proposition 1.6 or Theorem 1.8 for $p=3$ (for instance that $\varphi$ is stably $\mathrm{CH}^{2}$-elementary), and assume further that there is no F-algebra homomorphism $C_{0}\left(\left\langle d_{ \pm} \varphi\right\rangle \perp \psi\right) \rightarrow D$. Then

$$
N E^{3}\left(X_{\varphi \perp \psi}\right)=0 \text {. }
$$

Proof. Let $K, \tilde{\psi}$ be as in Proposition 1.6 or in Theorem 1.8. By this proposition or theorem and Lemma 1.2, it suffices to show that $N E K^{3}\left(X_{\varphi_{K} \perp\langle\tilde{\psi}\rangle}\right)=0$. By Proposition 1.12, this will be the case if we can show that ind $C_{0}\left(\varphi_{K}\right)=$ ind $C_{0}\left(\varphi_{K}\left(\sqrt{d_{ \pm}\left(\varphi_{K} \perp\langle\tilde{\psi}\rangle\right)}\right)\right.$. But $K\left(\sqrt{d_{ \pm}\left(\varphi_{K} \perp\langle\tilde{\psi}\rangle\right)}\right)=F\left(X_{\left\langle d_{ \pm} \varphi\right\rangle \downarrow}\right)$, so the result follows from the index reduction Theorem [24].

We are now ready to refine $[21$, Th. 6.1$]$ somewhat. 
1.14. Lemma. Let $\varphi \in I^{2} F \backslash I^{3} F$ be an 8-dimensional form, and $a \in F^{*}$. Then $\varphi \perp<a>$ is $\mathrm{CH}^{3}$-elementary.

Proof. Since $\varphi \notin I^{3} F$, it is $C H^{2}$-elementary; the claim then follows from Corollary 1.11.

1.15. Proposition. Let $\tau \in I^{2} F \backslash I^{3} F$ be an anisotropic 8-dimensional form, $\psi$ be another form of dimension $\geq 2$ and $q=\tau \perp \psi$. Then $q$ is $\mathrm{CH}^{3}$ elementary, except perhaps if $\tau_{F\left(X_{\psi}\right)}$ is similar to an anisotropic Pfister form. The latter happens exactly if $c(\tau)=c(\rho)$ for some $\rho \in P_{2}(F)-\{0\}$ and

(i) either $\psi$ is a neighbour of $\rho$;

(ii) or $\psi \simeq c\langle 1,-a\rangle$ with $\langle 1,-a\rangle \leq \rho$ and $\tau_{F(\sqrt{a})}$ is anisotropic.

In particular, if either ind $c(\tau) \geq 4$ or $\operatorname{dim} \psi=4, \psi \notin I^{2} F$, then $q$ is $\mathrm{CH}^{3}$-elementary.

Proof. By Examples 1b) and 2c) after Definition 1.3, $\tau$ is stably $\mathrm{CH}^{1}$ elementary and stably $\mathrm{CH}^{2}$-elementary in codimension 1 . We want to apply Theorem 1.8; for this, we need $\tau_{F\left(X_{\psi}\right)}$ to be $C H^{2}$-elementary. This is guaranteed by the assumptions (see Example 2a) after Definition 1.3). We then get an isomorphism

$$
C H^{3}\left(X_{q}\right)_{\mathrm{tors}} \simeq C H^{3}\left(X_{\tau \perp\langle\tilde{\psi}\rangle}\right)_{\mathrm{tors}} .
$$

Since the field of definition $L$ of $\tilde{\psi}$ is purely transcendental over $F, \tau_{L}$ is not in $I^{3} L$; by Lemma 1.14, the right-hand-side is 0 .

There remains to show that the condition of Proposition 1.15 happens exactly in cases (i) and (ii). The condition $c(\tau)_{F\left(X_{\psi}\right)}=0$ implies that ind $c(\tau) \leq 2$, hence (since $\left.\tau \notin I^{2} F\right) c(\tau)=(a, b) \neq 0$ for some $a, b$. Let $\rho=\left\langle a, b \gg\right.$. Then $\rho_{F\left(X_{\psi}\right)} \sim 0$, hence $\psi$ is similar to a subform of $\rho$. If $\psi$ is a neighbour of $\rho$, then $\tau_{F\left(X_{\psi}\right)}$ is anisotropic by [23, Th. 4]. Otherwise, $\operatorname{dim} \psi=2$ and we must impose $\tau_{F\left(\sqrt{d_{ \pm} \psi}\right)} \nsim 0$. Conversely, it is clear that (i) or (ii) implies the condition of Proposition 1.15.

1.16. Corollary. Let $q$ be an anisotropic form of dimension 10 , with $d=$ $d_{ \pm} q \neq 1$, and let $E=F(\sqrt{d})$. Assume that $q_{E}$ is isotropic, but that $\left(q_{E}\right)_{\mathrm{an}}$ is not similar to a nonzero Pfister form. Then $\mathrm{CH}^{3}\left(X_{q}\right)_{\text {tors }}=0$.

Proof. The fact that $i\left(q_{E}\right)>0$ implies that $q \simeq \tau \perp\langle a,-a d\rangle$ for some $\tau \in I^{2} F$ and $a \in F^{*}$. Then $q_{E} \sim \tau_{E}$, and the second condition is just a reformulation of the condition in Proposition 1.15. 
1.17. Proposition. Let $q$ be a form of dimension $\geq 9$ containing an anisotropic Albert form. Suppose that $\mathrm{CH}^{3}\left(X_{q}\right)_{\text {tors }} \neq 0$. Then:

(i) $\operatorname{dim} q \leq 12$

(ii) If $\operatorname{dim} q=12$, then $q \in I^{3} F$.

(iii) If $\operatorname{dim} q=11$, then $q \perp\left\langle-d_{ \pm} q\right\rangle \in I^{3} F$.

(iv) If $\operatorname{dim} q=10$, then two cases are possible:

- $d_{ \pm} q \neq 1$. Then $q$ is of the form $\lambda \perp a \lambda \perp b\langle 1,-d\rangle$, with $\operatorname{dim} \lambda=4$ and $d=d_{ \pm} q=d_{ \pm} \lambda$.

- $d_{ \pm} q=1$. Then ind $c(q)=2$.

(v) $\operatorname{dim} q=9$. Then ind $c(q)=2$.

Proof. Write $q=\varphi \perp \psi$, where $\varphi$ contains an anisotropic Albert form and $\operatorname{dim} \varphi=7$. By Example 2b) after Definition 1.3, $\varphi$ is stably $\mathrm{CH}^{2}$ elementary.

Let $D$ be the division algebra similar $C_{0}(\varphi)$ : then $D$ is a biquaternion algebra. By Proposition 1.13, if $C H^{3}\left(X_{q}\right)_{\text {tors }} \neq 0$, then there must exist an $F$-algebra homomorphism $A \rightarrow D$, where we set $C_{0}\left(\left\langle d_{ \pm} \varphi\right\rangle \perp \psi\right)=A$ for simplicity. This first gives a condition on $\operatorname{dim} q$ :

- If $\operatorname{dim} \psi$ is even, then $A$ is central simple. We then must have $\operatorname{dim} A \leq$ $\operatorname{dim} D$, that is, $\operatorname{dim} \psi \leq 4$, i.e. $\operatorname{dim} q \leq 11$.

- If $\operatorname{dim} \psi$ is odd, then $A$ is either simple with centre $F\left(\sqrt{-d_{ \pm} \varphi d_{ \pm} \psi}\right)$, or a product of two isomorphic simple algebras, and then $-d_{ \pm} \varphi d_{ \pm} \psi$ is a square. In the first case, we must again have $\operatorname{dim} \psi \leq 4$; in the second one, we must have $\operatorname{dim} \psi \leq 5$. In both cases, this gives $\operatorname{dim} q \leq 12$.

Let us now look at the extreme cases. It is useful to note that $d_{ \pm} q=$ $(-1)^{\operatorname{dim} \psi} d_{ \pm} \varphi d_{ \pm} \psi$ and that $c(q)=c(\varphi)+c\left(d_{ \pm} \varphi \perp \psi\right)$.

- If $\operatorname{dim} q=12$, then the above discussion shows that $d_{ \pm} q=1$; moreover, $D \simeq A$, which gives $c(q)=0$. Hence $q \in I^{3} F$.

- If $\operatorname{dim} q=11$, then we get similarly $c(q)=0$, hence $c\left(q \perp\left\langle-d_{ \pm} q\right\rangle\right)=0$ and $q \perp\left\langle-d_{ \pm} q\right\rangle \in I^{3} F$.

- If $\operatorname{dim} q=10$ and $d_{ \pm} q=d \neq 1$, then $E=F(\sqrt{d})$ embeds into $D$ and $A$ is isomorphic to its commutant. Therefore, $D_{E} \sim A$ and $c\left(q_{E}\right)=0$. Write $q \simeq \gamma \perp \rho$, where $\gamma$ is an anisotropic Albert form and $\operatorname{dim} \rho=4$. Then $d_{ \pm} \rho=d$ and $c\left(\gamma_{E}\right)=c\left(\rho_{E}\right)$, hence $\gamma_{E}$ is isotropic, i.e. $\gamma \simeq \lambda \perp a\langle 1,-d\rangle$ for suitable $\lambda, a$. But $\lambda_{E}$ and $\rho_{E}$ are conjugate 4-dimensional forms ${ }^{1}$, hence are similar by [10, Cor. 2.10]; a theorem of Wadsworth [34, Th. 7] then implies that $\lambda$ and $\rho$ are similar.

\footnotetext{
1 Recall that two forms $\varphi, \psi$ are conjugate if $\varphi \perp-\psi$ is similar to a (possibly trivial) Pfister form, $c f$. [22, Def. 8.7].
} 
Finally, let us look at the cases $\operatorname{dim} q=10, d_{ \pm} q=1$ and $\operatorname{dim} q=9$. In the first case, $A=B \times B$ and $B$ injects into $D$, hence ind $c(q)=2$. In the second case, $A$ is a quaternion algebra and injects into $D$, hence ind $c(q)=2$ too.

\subsection{Remarks.}

1. The first case in (iv) of Proposition 1.17 is a special case of the exceptional case in Corollary 1.16.

2. We know by Karpenko [21] that, in fact, $C H^{3}\left(X_{q}\right)_{\text {tors }}=0$ for $\operatorname{dim} q>12$ whether $q$ contains an anisotropic Albert form or not.

\section{Proof of Theorems 1 and 2}

In this section we assume that $F$ has characteristic 0 .

2.1. Definition. An anisotropic quadratic form $q$ of dimension $\geq 7$ is exceptional if

- $q$ is a neighbour of a 3-fold Pfister form, or

- writing $q_{F\left(X_{q}\right)} \simeq \mathbf{H} \perp q^{\prime}$, the form $q^{\prime}$ is an anisotropic neighbour of a 3-fold Pfister form.

2.2. Proposition. Let $\operatorname{dim} q \geq 7$ and $X=X_{q}$.

a) The map $H^{5}(F, \mathbf{Q} / \mathbf{Z}(3)) \stackrel{\sim}{\rightarrow} H^{6}(F, \mathbf{Z}(3)) \rightarrow H^{6}(X, \mathbf{Z}(3))$ is injective. b) Assume $q$ is anisotropic and not exceptional. Then the cycle map $\mathrm{cl}^{3}$ : $C H^{3}(X) \rightarrow H^{6}(X, \mathbf{Z}(3))$ induces a map

$$
{ }_{2} C H^{3}(X) \rightarrow H^{5}(F, \mathbf{Q} / \mathbf{Z}(3))
$$

still denoted by $\mathrm{cl}^{3}$.

Proof. Recall from [16] the spectral sequence

$$
E_{2}^{p, q}=H_{\mathrm{et}}^{p-q}\left(F, C H^{q}\left(X_{s}\right) \otimes \mathbf{Z}(3)\right) \Rightarrow H^{p+q}
$$

with $H^{p+q}$ mapping to $H^{p+q}(X, \mathbf{Z}(3))$ by an isomorphism if $p+q \leq 6$ and an injection if $p+q=7$. It gives a (decreasing) filtration $F^{q} H^{6}(X, \mathbf{Z}(3))$ on $H^{6}(X, \mathbf{Z}(3))$ in four steps, whose associated graded are subquotients of the $E_{2}$-terms

$$
\begin{aligned}
& E_{2}^{6,0}=H^{6}(F, \mathbf{Z}(3)) \\
& E_{2}^{5,1}=H^{4}\left(F, C H^{1}\left(X_{s}\right) \otimes \mathbf{Z}(2)\right) \\
& E_{2}^{4,2}=H^{2}\left(F, C H^{2}\left(X_{s}\right) \otimes \mathbf{Z}(1)\right) \\
& E_{2}^{3,3}=H^{0}\left(F, C H^{3}\left(X_{s}\right)\right) .
\end{aligned}
$$


By Hilbert 90 in weight 1 , the third $E_{2}$-term is 0 . Similarly, by Hilbert 90 in weight 2 , one has $E_{2}^{4,1}=0$. So the only possibly nonzero differential hitting $E_{2}^{6,0}=E_{3}^{6,0}$ is

$$
d_{3}^{3,2}: E_{3}^{3,2} \rightarrow E_{3}^{6,0}
$$

But by [16, Prop. 6.5 a)], $E_{2}^{3,2}=E_{\infty}^{3,2}$ provided the map $C H^{2}(X) \rightarrow$ $C H^{2}\left(X_{s}\right)$ is surjective; this is the case when $\operatorname{dim} X>4$, i.e. when $\operatorname{dim} q>6$ (cf. loc. cit., Remark 6.6). This proves a).

Let now $e$ be an element of ${ }_{2} C H^{3}(X)$, and let $\alpha=c l^{3}(e)$ be its image in $H^{6}(X, \mathbf{Z}(3))$ under the cycle map. Since $C H^{3}\left(X_{s}\right)$ is torsion-free, its image via the edge homomorphism $H^{6}(X, \mathbf{Z}(3)) \rightarrow C H^{3}\left(X_{s}\right)^{G_{F}}$ is 0 . Hence $\alpha \in F^{1} H^{6}(X, \mathbf{Z}(3))$. Let $\bar{\alpha}$ be its image in $E_{2}^{5,1}=H^{4}\left(F, C H^{1}\left(X_{s}\right) \otimes \mathbf{Z}(2)\right)$. Since $\operatorname{dim} X>2, C H^{1}\left(X_{s}\right)=\mathbf{Z} h$ (where $h$ is a hyperplane section) and $H^{4}\left(F, C H^{1}\left(X_{S}\right) \otimes \mathbf{Z}(2)\right)$ is canonically isomorphic to $H^{4}(F, \mathbf{Z}(2)) \stackrel{\sim}{\leftarrow}$ $H^{3}(F, \mathbf{Q} / \mathbf{Z}(2))$.

First suppose $q$ isotropic. Write $q \simeq \mathbf{H} \perp q^{\prime}$, and let $Y$ be the projective quadric with equation $q^{\prime}=0$. Then there is an isomorphism $C H^{3}(X) \simeq$ $C H^{2}(Y)$. If $q^{\prime}$ is not a neighbour of an anisotropic 3-fold Pfister form, then ${ }_{2} C H^{2}(Y)=0$ [19, Th. 6.1], hence ${ }_{2} C H^{3}(X)=0$ and in particular $\bar{\alpha}=0$.

Suppose now $q$ anisotropic, and let $K=F(X)$. We have a commutative diagram

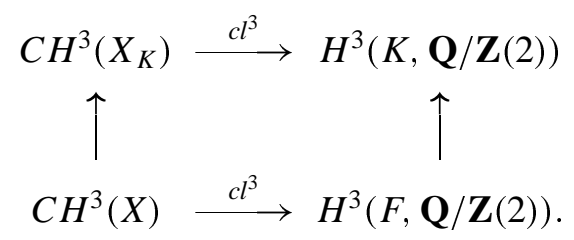

By Arason's theorem [1, Satz 5.6] (and the Merkurjev-Suslin theorem), the map $H^{3}(F, \mathbf{Q} / \mathbf{Z}(2)) \rightarrow H^{3}(K, \mathbf{Q} / \mathbf{Z}(2))$ is injective, unless $q$ is a neighbour of a 3 -fold Pfister form. By the isotropic case, $\bar{\alpha}_{K}=0$, hence (under the assumptions of the proposition) $\bar{\alpha}=0$ and $\alpha \in F^{2} H^{6}(X$, $\mathbf{Z}(3))=F^{3} H^{6}(X, \mathbf{Z}(3))$. This proves $\left.\mathrm{b}\right)$, noting that $F^{3} H^{6}(X, \mathbf{Z}(3))=$ $H^{5}(F, \mathbf{Q} / \mathbf{Z}(3))$.

2.3. Proposition. Let $\operatorname{dim} q \geq 7$. Assume $q$ is anisotropic and is not a neighbour of a 3-fold Pfister form. Then $q$ is exceptional if and only if it is of one of the following three types:

a) $\operatorname{dim} q=7:$ ind $c(q)=2$.

b) $\operatorname{dim} q=8: d=d_{ \pm} q \neq 1$ and $q_{F(\sqrt{d})} \in G P_{3}(F(\sqrt{d})) \backslash\{0\}$.

c) $\operatorname{dim} q=9: q \simeq \pi \perp\langle d\rangle$ with $\pi \in G P_{3}(F)$. 
Proof. We must have $7 \leq \operatorname{dim} q \leq 10$. Let $X=X_{q}$. If $\operatorname{dim} q=10$, then $q_{F(X)} \in I^{3} F(X)$, hence $d_{ \pm} q=1, c(q)=0$ since $H^{i} F \rightarrow H^{i} K$ is injective for $i=1,2$. But then $q$ is isotropic by [28, Satz 14], contradiction. So $\operatorname{dim} q \leq 9$.

If $\operatorname{dim} q=9$, we see similarly that $q \perp\left\langle-d_{ \pm} q\right\rangle \in I^{3} F$, hence that $q$ represents its discriminant $d=d_{ \pm} q$. Writing $q \simeq \pi \perp\langle d\rangle$, we find $\pi \in I^{3} F$, hence $\pi \in G P_{3}(F)$. Conversely, any form of this type is obviously exceptional.

If $\operatorname{dim} q=8$, write $d=d_{ \pm} q$ and $K=F(X)$. Note that $d /=1$ since $d=d_{ \pm}\left(q_{K}\right)_{\text {an }}$ and $\left(q_{K}\right)_{\text {an }}$ is a 6-dimensional Pfister neighbour. Let $E=$ $F(\sqrt{d})$. By assumption, $q_{K E} \sim 0$, hence $q_{E} \in G P_{3}(E)$. But if $q_{E} \sim 0$, then $q$ is divisible by $\langle 1,-d\rangle$, hence $q \in I^{2} F$, contadicting $d \neq 1$. Conversely, suppose that $q$ satisfies the condition of b). Then, for any $a \in F^{*}, c(q \perp$ $-a\langle 1,-d\rangle)$ is of the form $(b, d)$ for some $b$. By changing $a$ into $a b$, we can arrange that $q \perp-a\langle 1,-d\rangle \in I^{3} F$. Then, applying [28, Satz 14] again, we find $(q \perp-a\langle 1,-d\rangle)_{\text {an }}=: \pi \in G P_{3}(F)$. Suppose that $i\left(q_{K}\right)>1$. Then $\pi_{K} \sim 0$; by the subform theorem, this forces $\pi$ to be similar to $q$, which is absurd. Hence $i\left(q_{K}\right)=1$ and $\left(q_{K}\right)_{\text {an }}$ is a neighbour of $\pi_{K}$.

Finally, let $\operatorname{dim} q=7$. Then ind $c\left(q_{F(X)}\right)=2$, hence ind $c(q)=2$ by the index reduction theorem. Conversely, suppose that $q$ satisfies this condition. Then $i\left(q_{F(X)}\right)=1$ : otherwise, by [15, Prop. 3 (ii)], $q_{1}=\left(q_{F(X)}\right)$ an would be defined over $F$ and $q \perp-q_{1}$ would be similar to a Pfister form, absurd. Since ind $c\left(q_{1}\right)=2, q_{1}$ is a Pfister neighbour.

\subsection{Proposition. For any exceptional form $q$, one has Coker $\eta^{4}=0$.}

Proof. By [17, Th. 6 (3)], we may suppose that $q$ is not a neighbour of a 3 -fold Pfister form. Then $q$ is classified by Proposition 2.3. If $\operatorname{dim} q=7$, by Proposition 2.3 a), $q$ is a subform of an 8-dimensional form $q^{\prime}$ in $I^{2} F$ with Clifford invariant of index 2. Then $q$ and $q^{\prime}$ are equivalent in the sense that $q_{F\left(X_{q}\right)}^{\prime}$ and $q_{F\left(X_{q^{\prime}}\right)}$ are isotropic (in the latter case, note that $q_{F\left(X_{q^{\prime}}\right)}$ represents its discriminant, hence is isotropic, see end of proof of Proposition 2.3). By [17, Prop. 2.5], Coker $\eta_{q}^{4} \simeq$ Coker $\eta_{q^{\prime}}^{4}$ and, by [17, Th. 6 (3)], Coker $\eta_{q^{\prime}}^{4}=0$. If $\operatorname{dim} q=9, q$ is a Pfister neighbour by Proposition $2.3 \mathrm{c}$ ), hence Coker $\eta^{4}=0$ again by [17, Th. 6 (3)].

Finally, assume that $\operatorname{dim} q=8$. Let $X=X_{q}$. We shall apply results from [19] to show that $C H^{3}(X)_{\text {tors }}=\mathbf{Z} / 2$, but that the motivic cycle map is injective in codimension 3.

By [19, Cor. 4.5], for the first claim it suffices to prove that $G^{3} K(X)_{\text {tors }}=$ $\mathbf{Z} / 2$, where $G^{*} K(X)$ is the associated graded of the topological filtration on $K_{0}(X)$. We note that $q \notin I^{2} F$; by [19, Th. 3.10], $G^{p} K(X)_{\text {tors }}$ is of order at most 2 and $\left|G^{*} K(X)_{\text {tors }}\right|=2^{s}$, where $s$ is such that $C_{0}(q) \simeq M_{2^{s}}(D)$ with 
$D$ a division algebra. Here, one computes easily from Proposition $2.3 \mathrm{~b}$ ) that $s=3$. Moreover, $G^{p} K(X)_{\text {tors }}=0$ for $p=0,1,2,6$ by [19, Prop. 2.4, Prop. 2.6 and Th. 6.1]. It follows that $G^{p} K(X)_{\text {tors }}=\mathbf{Z} / 2$ for $p=3,4,5$. More precisely, we have $G^{p} K(X)_{\text {tors }}=\mathbf{Z} / 2 \bar{l}_{6-p-1}$ for $p=3,4$, 5, where $l_{0}, l_{1}, l_{2}$ are the $K_{0}$-classes defined by Karpenko in [19, (2.1) and (3.7)] and $\bar{l}_{i}$ are their images in the associated graded.

Consider now the situation over $K=F(X)$. Then $i\left(q_{K}\right)=1$, hence, over $K$, we still have $\operatorname{dim} l_{2}>\operatorname{dim} l_{1}>1$. Therefore $l_{1}$ and $l_{2}$ still define torsion classes in $G^{*} K\left(X_{K}\right)$, of codimensions $\leq 4$. But $G^{p} K\left(X_{K}\right)$ is torsion-free for $p \leq 2$, so we must have $\operatorname{codim}\left(l_{2}\right)=3$, $\operatorname{codim}\left(l_{1}\right)=4$. This argument shows in particular that the map $C H^{3}(X) \rightarrow C H^{3}\left(X_{K}\right)$ is injective on torsion.

Write $q_{K} \simeq \varphi \perp \mathbf{H}$, where $\varphi$ is a 6-dimensional Pfister neighbour. Let $Y$ be the quadric with equation $\varphi=0$. By [16, Prop. 8.1], there is a commutative diagram

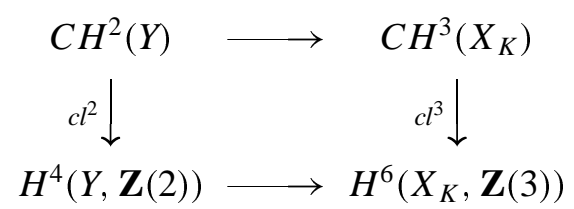

in which the top horizontal map is an isomorphism and the bottom one is a split injection. Moreover, $c l^{2}$ is injective by [9, Th. 1.1] or [16, diagram in 5.3].

By [19, Th. 6.1], $C H^{2}(Y)_{\text {tors }}=\mathbf{Z} / 2$, hence $C H^{3}\left(X_{K}\right)_{\text {tors }}=\mathbf{Z} / 2$. Letting $e^{\prime}$ denote its generator, the diagram shows that $c l^{3}\left(e^{\prime}\right) \neq 0$. Since by the above $e^{\prime}$ is the image of the torsion element $e$ of $C H^{3}(X)$, this shows that $c l^{3}(e) \neq 0$ as well. Then (1) shows that Coker $\eta^{4}=0$.

2.5. Lemma. Let q be a form of dimension 10 in $I^{2} F$ such that ind $c(q)=2$. Then the cycle class map

$$
c l^{3}: C H^{3}\left(X_{q}\right)_{\text {tors }} \rightarrow H^{6}\left(X_{q}, \mathbf{Z}(3)\right)
$$

is identically 0.

Proof. Let $D$ be the quaternion algebra whose Brauer class is $c(q)$ and let $E$ be a maximal commutative subfield of $D$. The quadratic form $q_{E}$ is in $I^{3} E$, hence $q_{E} \simeq \varphi \perp \mathbf{H}$, where $\varphi \in G P_{3}(E)$. By [18, Lemma 4.2], we get easily $c l^{3}(e)=c l^{1}(h) \cdot \pi^{*} \beta e^{3}(\varphi)$, where $e$ is the (nonzero) generator of $C H^{3}\left(X_{q_{E}}\right)_{\text {tors }} \simeq C H^{2}(Y)_{\text {tors }}$, with $Y=X_{\varphi}$. But the quadratic extension $E / F$ is excellent, hence $\varphi$ is defined over $F$. It follows that

$$
c l^{3}\left(N_{E / F} e\right)=\operatorname{Cor}_{E / F} c l^{3}(e)=\operatorname{Cor}_{E / F}\left(c l^{1}(h) \cdot \pi^{*} \beta e^{3}(\varphi)\right)=0 .
$$

By Lemma 1.2 and Proposition 1.9, the map $N_{E / F}: C H^{3}\left(X_{q_{E}}\right)_{\text {tors }} \rightarrow$ $\mathrm{CH}^{3}\left(\mathrm{X}_{q}\right)_{\text {tors }}$ is surjective. This concludes the proof of Lemma 2.5. 
Proof of Theorem 1. By Proposition 2.2, for $q$ non exceptional (1) refines into an exact sequence

$$
0 \rightarrow \operatorname{Coker} \eta^{4} \rightarrow C H^{3}(X)_{\text {tors }} \rightarrow H^{5}(F, 3) .
$$

In particular, b) follows from a) and to prove a), we have to show that the right map is 0 for $q$ as in the theorem. By functoriality, it is enough to do this for $\operatorname{dim} q=9$. By Proposition 1.10 and functoriality again, it is enough to deal with $\operatorname{dim} q=10, q \in I^{2} F$. Applying now Proposition 1.9, we can reduce to the case where ind $c(q)=2$. But this case is covered by Lemma 2.5.

Proof of Theorem 2. This is a case-by-case check collecting the previous results. Assertion (i) follows from Propositions 2.4 and 2.3. Assertion (ii) follows from [17, Theorem 6 (3)] and Propositions 2.4 and 2.3 (note that the proof of Proposition 2.3 b) shows that, if $\operatorname{dim} q=8, d=d_{ \pm} q \neq 1$ and $q_{F(\sqrt{d})}$ is similar to a Pfister form, then this Pfister form is necessarily nonzero). Assertion (iii) follows from Propositions 2.4 and 2.3 when $c(q)=1$. Otherwise, it follows from Theorem 1, Lemma 1.14 and Proposition 1.17 (v). Assertions (iv), (v) and (vi) follow from Theorem 1, Propositions 1.15 and 1.17. Assertion (vi) follows from [17, Theorem 6 (3)]. Finally, the last statement follows from Theorem 1 and [20, Th. 2.4].

\section{Unramified cohomology of Pfister quadrics}

3.1. Proof of Theorem 3. In this subsection, we occasionally refer to a preliminary version of [27] which was kindly communicated to us by A. Vishik. We don't use results from [27], but just point out that some of our results will independently appear in this paper (and hence were found by the authors earlier than us).

By [17, Prop. 2.5 (c)], Coker $\tilde{\eta}^{m+1}$ does not depend on the choice of the neighbour $\psi$; henceforth we choose $\psi$ with $\operatorname{dim} \psi=2^{n-1}+1$. Then $\operatorname{dim} X=2^{n-1}-1$. On the other hand, by [17, Prop. 2.5 (b)], the group Coker $\tilde{\eta}^{m+1}$ is isomorphic to

$$
\operatorname{Coker}\left(\eta^{m+1}: H^{m+1}\left(F, \mathbf{Q}_{2} / \mathbf{Z}_{2}(m)\right) \rightarrow H_{\mathrm{nr}}^{m+1}\left(F(X) / F, \mathbf{Q}_{2} / \mathbf{Z}_{2}(m)\right)\right) .
$$

Recall from [33] the simplicial scheme $\check{C}(X)$, with $\check{C}(X)_{p}=X^{p+1}$, the faces and degeneracies being the obvious projections and partial diagonals. In [16, Th. A.1], we proved that Coker $\eta^{m+1}$ is isomorphic to a subgroup of $H^{m+3}\left(\check{C}(X), \mathbf{Z}_{(2)}(m)\right)$, where the latter group is weight $m$ motivic cohomology of $\check{C}(X)$ (it is denoted with an index $B$ in [33]). Theorem 3 therefore follows from the more precise statement: 
3.1. Proposition. Let $\psi$ be a Pfister neighbour of dimension $2^{n-1}+1$ and $X$ be the associated Pfister quadric. Then the group $H^{m+3}\left(\check{C}(X), \mathbf{Z}_{(2)}(m)\right)$ is 0 for all $m \geq 0$.

This proposition is also a special case of a theorem which will appear in [27]. The proof faithfully follows the method of Voevodsky [33] in his proof of the Milnor conjecture. It is slightly simpler than the proof in the version of [27] we saw, but they prove more than what we need for our purposes. In any case we don't claim any particular originality in finding the argument. All unexplained notation should be found in [33].

Proof. Recall from [33, §3.2] the stable homotopy category of schemes $\mathcal{S H}(F)$. For any simplicial scheme $X_{\bullet}$ over $F$, there is an associated object

$$
\Sigma_{T}^{\infty}(X)_{+} \in \mathcal{S H}(F) .
$$

By [33, Th. 3.12], the group $H^{p}\left(X_{\bullet}, \mathbf{Z}_{(2)}(q)\right)$ coincides with the group

$$
H^{p, q}\left(X_{\bullet}, \mathbf{Z}_{(2)}\right)=\operatorname{Hom}_{\mathcal{S H}}\left(\Sigma_{T}^{\infty}\left(X_{\bullet}\right)_{+}, S^{p, q} \wedge \mathbf{H}_{\mathbf{Z}_{(2)}}\right)
$$

where $\mathbf{H}_{\mathbf{Z}_{(2)}}$ is the motivic Eilenberg-Mac Lane spectrum. Similarly when replacing $\mathbf{Z}_{(2)}$ by $\mathbf{Z} / 2$. For such an $X_{\bullet}$, we denote as in [33, §3.3] by $\tilde{H}^{p, q}\left(X_{\bullet}, \mathbf{Z}_{(2)}\right)$ the group $H^{p, q}\left(\tilde{X}_{\bullet}, \mathbf{Z}_{(2)}\right)$ where $\tilde{X}_{\bullet}$ is the homotopy fibre of the natural morphism

$$
\Sigma_{T}^{\infty}\left(X_{\bullet}\right)_{+} \rightarrow S^{0}
$$

so that we have long exact sequences

$$
\begin{aligned}
\cdots \rightarrow \tilde{H}^{p, q}\left(X_{\bullet}, \mathbf{Z}_{(2)}\right) & \rightarrow H^{p}\left(X_{\bullet}, \mathbf{Z}_{(2)}(q)\right) \\
& \rightarrow H^{p}\left(F, \mathbf{Z}_{(2)}(q)\right) \rightarrow \tilde{H}^{p+1, q}\left(X_{\bullet}, \mathbf{Z}_{(2)}\right) \rightarrow \ldots
\end{aligned}
$$

and similarly with $\mathbf{Z} / 2$ coefficients. Finally, recall from [33, §3.3] the motivic Steenrod operations

$$
Q_{i}: H^{p, q}(S, \mathbf{Z} / 2) \rightarrow H^{p+2^{i+1}-1, q+2^{i}-1}(S, \mathbf{Z} / 2)
$$

which are natural in $S \in \mathcal{S H}(F)$. Since $\operatorname{dim} X=2^{n-1}-1$, by [33, Th. 3.25 and Lemma 4.11], the sequence

$$
\begin{aligned}
\ldots \stackrel{Q_{i}}{\longrightarrow} \tilde{H}^{*, *}(\check{C}(X), \mathbf{Z} / 2) \stackrel{Q_{i}}{\longrightarrow} \tilde{H}^{*, *}(\check{C}(X), \mathbf{Z} / 2) \\
\stackrel{Q_{i}}{\longrightarrow} \tilde{H}^{*, *}(\check{C}(X), \mathbf{Z} / 2) \stackrel{Q_{i}}{\longrightarrow} \ldots
\end{aligned}
$$

is exact for all $i \leq n-1$. As in [33], we shall use this result only up to $i=n-2$.

We now follow faithfully the computations of [33, §4], only replacing the group $H^{n+1, n}(\check{C}(X), \mathbf{Z} / 2)$ by $H^{m+3, m}(\check{C}(X), \mathbf{Z} / 2)$. For $j \leq n-1$, let 
us examine the effect of the operation $Q_{j} \cdots Q_{1}$ on this group. Its image is contained in

$$
H^{2^{j+2}+m-j-1,2^{j+1}+m-j-2}(\check{C}(X), \mathbf{Z} / 2) .
$$

The operation $Q_{j+1}$ reaches this group from

$$
H^{m-j, m-j-1}(\check{C}(X), \mathbf{Z} / 2) .
$$

By [33, Cor. 2.13(1)], the latter group injects into $H_{L}^{m-j}(\check{C}(X), \mathbf{Z} / 2(m-$ $j-1)$, which in turn is isomorphic to $H_{L}^{m-j}(F, \mathbf{Z} / 2(m-j-1))$ by loc. cit., Prop. 2.7. The latter group is 0 by loc. cit., Th. 4.1. By the exactness mentioned above, we conclude that $Q_{j+1}$ is injective on $H^{2^{j+2}+m-j-1,2^{j+1}+m-j-2}(\check{C}(X), \mathbf{Z} / 2)$ for all $j \in[0, n-2]$, hence $Q_{n-2} \cdots Q_{1}$ is injective on $H^{m+3, m}(\check{C}(X), \mathbf{Z} / 2)$. Moreover, $H^{p, q}\left(\check{C}(X), \mathbf{Z}_{(2)}\right)$ is naturally a subgroup of $H^{p, q}(\check{C}(X), \mathbf{Z} / 2)$ for $p>q$ and these subgroups are preserved by the operations $Q_{i}$ [33]. It therefore suffices to show that

$$
\begin{aligned}
H^{2^{n}+m-n+1,2^{n-1}+m-n}\left(\check{C}(X), \mathbf{Z}_{(2)}\right) \\
\quad=H^{2^{n}+m-n+1}\left(\check{C}(X), \mathbf{Z}_{(2)}\left(2^{n-1}+m-n\right)\right)=0 .
\end{aligned}
$$

To do this, we compute the latter group by using the exact triangle of [33, Th. 4.4]

$$
\begin{aligned}
M\left(\check{C}(X)\left(2^{n-1}-1\right)\left[2^{n}-2\right] \rightarrow M_{\psi}\right. & \rightarrow M(\check{C}(X)) \\
& \rightarrow M\left(\check{C}(X)\left(2^{n-1}-1\right)\left[2^{n}-1\right] .\right.
\end{aligned}
$$

Here this triangle is defined in the category $D M_{-}^{e f f}(F)$ of [32], and $M_{\psi}$ is the Rost motive associated to $\psi$ [29]. We get an exact sequence

$$
\begin{aligned}
H^{m-n+2}\left(\check{C}(X), \mathbf{Z}_{(2)}(m-n+1)\right) & \rightarrow H^{2^{n}+m-n+1}\left(\check{C}(X), \mathbf{Z}_{(2)}\left(2^{n-1}+m-n\right)\right) \\
& \rightarrow H^{2^{n}+m-n+1}\left(M_{\psi}, \mathbf{Z}_{(2)}\left(2^{n-1}+m-n\right)\right) .
\end{aligned}
$$

As seen above, the left group is 0 . The right one is a direct summand of

$$
H^{2^{n}+m-n+1}\left(X, \mathbf{Z}_{(2)}\left(2^{n-1}+m-n\right)\right) .
$$

But $2^{n}+m-n+1-\left(2^{n-1}+m-n\right)>2^{n-1}-1=\operatorname{dim} X$, so this group is also 0 by [33, Cor. 2.4]. 
The following corollary was pointed out by A. Vishik.

3.2. Corollary. For any quadric $X$, the injection of [16, Th. A.1]

$$
\text { Coker } \eta^{m+1} \rightarrow H^{m+3}\left(\check{C}(X), \mathbf{Z}_{(2)}(m)\right)
$$

is an isomorphism.

Proof. The precise statement of loc. cit. is that there is an exact sequence

$$
0 \rightarrow \operatorname{Coker} \eta^{m+1} \rightarrow H^{m+3}\left(\check{C}(X), \mathbf{Z}_{(2)}(m)\right) \stackrel{\varphi_{X}}{\longrightarrow} H^{m+2}\left(F, \mathbf{Q}_{2} / \mathbf{Z}_{2}(m)\right)
$$

which is contravariant in $X$. In particular, let $C \subseteq X$ be a smooth subconic of $X$ (obtained by taking some plane section). By Proposition 3.1, $H^{m+3}\left(\breve{C}(C), \mathbf{Z}_{(2)}(m)\right)=0$, hence $\varphi_{C}=0$. By functoriality, this implies $\varphi_{X}=0$.

3.3. Remark. In [16, Th. A.1], an isomorphism

$$
\operatorname{Ker} \eta^{m+1} \simeq H^{m+2}\left(\check{C}(X), \mathbf{Z}_{(2)}(m)\right)
$$

is also proven. The same arguments as above then yield an injection

$$
\operatorname{Ker} \eta^{m+1} \hookrightarrow H^{2^{n}+m-n}\left(\check{C}(X), \mathbf{Z}_{(2)}\left(2^{n-1}+m-n\right)\right)
$$

and an exact sequence

$$
\begin{aligned}
A_{0}\left(M_{\varphi}, K_{m-n+1}^{M}\right) & \rightarrow K_{m-n+1}^{M}(F) \\
& \stackrel{\alpha}{\rightarrow} H^{2^{n}+m-n}\left(\check{C}(X), \mathbf{Z}_{(2)}\left(2^{n-1}+m-n\right)\right) \rightarrow 0 .
\end{aligned}
$$

Most likely this corresponds to an exact sequence of [27]:

$$
A_{0}\left(X, K_{m-n+1}^{M}\right) \rightarrow K_{m-n+1}^{M}(F) \stackrel{\cdot e^{n}(\varphi)}{\longrightarrow} K_{m+1}^{M}(F) / 2 \rightarrow K_{m+1}^{M}(F(X)) / 2
$$

In any case, it implies for $m=n-1$ that $\operatorname{Ker} \eta^{n}=\operatorname{Ker} \eta_{2}^{n}$ has order $\leq 2$, which is sufficient to imply the second Milnor conjecture that $e^{n}$ : $I^{n} F / I^{n+1} F \rightarrow H^{n} F$ is a well-defined isomorphism [13, Remark p. 555]. This result is [27, 2.1]. F. Morel has recently given an independent proof of the bijectivity of $e^{n}$, using an analogue of the Adams spectral sequence converging to the stable homotopy of the motivic sphere [26]. 
3.2. Proof of Theorem 4. We may assume $F$ finitely generated. Then its virtual 2-cohomological dimension $v c d_{2}(F)$ is finite; similarly, $v c d_{2}(F(X))<+\infty$.

We shall use [33] and the fact that $e^{m}: I^{m} F / I^{m+1} F \rightarrow H^{m} F$ is a welldefined isomorphism for all $m$ and all $F$ [27], [26] and the above remark). By Theorem 3 and (the proof of) [17, Th. C.1], this implies that the natural map

$$
I^{m} F / I^{m+1} F \rightarrow I_{\mathrm{nr}}^{m}(F(X) / F) / I_{\mathrm{nr}}^{m+1}(F(X) / F)
$$

is surjective for all $m$, where $I_{\mathrm{nr}}^{m}(F(X) / F)=I^{m}(F(X) / F) \cap W_{\mathrm{nr}}(F(X) / F)$. It is therefore enough to prove that the map

$$
I^{m} F \rightarrow I_{\mathrm{nr}}^{m}(F(X) / F)
$$

is surjective for $m$ sufficiently large.

The following lemma is well-known, but we include it for lack of reference.

3.4. Lemma. Suppose that $c d_{2}(F)=d$. Then $I^{d+1} F=0$.

Proof. Let $\varphi$ be a $(d+1)$-fold Pfister form over $F$. Then $e^{d+1}(\varphi) \in$ $H^{d+1} F=0$, hence $\varphi \in I^{d+2} F$ and $\varphi=0$ by the Arason-Pfister theorem.

If $F(X)$ is not formally real the proof is finished since then $I_{\mathrm{nr}}^{m}(F(X) / F)=0$ for $m>c d_{2}(F(X))$ by Lemma 3.4. In the sequel of the proof, we are only concerned with the other case.

For a field $K$, let sper $K$ denote the space of orderings ("real spectrum") of $K$ : this is a compact totally disconnected topological space.

3.5. Lemma. Let $m>d=v c d_{2}(K)$. Then the signature map induces an isomorphism

$$
\text { sgn }: I^{m} K \stackrel{\sim}{\rightarrow} C\left(\operatorname{sper} K, 2^{m} \mathbf{Z}\right)
$$

where $C$ denotes the group of continuous functions.

Proof. Consider the field $L=K(\sqrt{-1})$. Then $c d_{2} L=d$ and hence $I^{d+1} L=0$ by Lemma 3.4. By [2, Lemma 3.6], the group $I^{d+1} K$ is then torsion-free, hence $s g n$ is injective on $I^{d+1} K$ by Pfister's theorem [31, Th. 7.3 p. 56]. Let $\xi \in C\left(\operatorname{sper} K, 2^{m} \mathbf{Z}\right)$. By [31, Th. 6.1, p. 128], there exist $n \geq 0$ and $q \in W(F)$ such that $\operatorname{sgn}(q)=2^{n} \xi$. Hence $\operatorname{sgn}(q) \in$ $C\left(\operatorname{sper} K, 2^{n+m} \mathbf{Z}\right)$. Further, for $m$ as above, we have isomorphisms [2] 


$$
I^{m} K / I^{m+1} K \stackrel{\bar{e}_{m}}{\longrightarrow} H^{m}(K, \mathbf{Z} / 2) \stackrel{h_{m}}{\longrightarrow} C(\operatorname{sper} K, \mathbf{Z} / 2),
$$

such that for $\psi \in I^{m} K, h_{m} \bar{e}_{m}([\psi]) \equiv \operatorname{sgn}(\psi)(\bmod 2)$.

Consider the form $2^{d+1} q \in I^{d+1} K$. We have $\operatorname{sgn}\left(2^{d+1} q\right) \in C(\operatorname{sper} K$, $2^{n+m+d+1} \mathbf{Z}$ ). By the isomorphisms (5), we see that $2^{d+1} q \in I^{n+m+d+1} K$. We have a commutative diagram

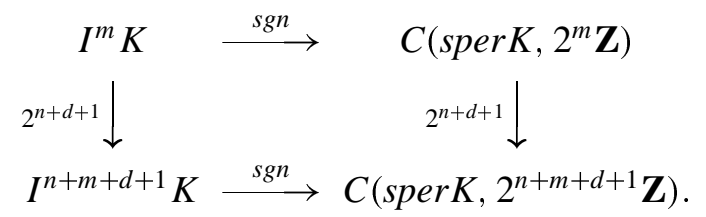

But the vertical arrows are isomorphisms since $m>d$ [2]. Hence $2^{d+1} q=$ $2^{n+d+1} \varphi$, for a form $\varphi \in I^{m} K$ such that $\operatorname{sgn}(\varphi)=\xi$, and the lemma is proved.

We can split sperF into a union of two clopen sets:

$$
\operatorname{sperF}=S_{a} \coprod S_{i}
$$

where

$$
\begin{aligned}
& S_{a}=\left\{v \in \operatorname{sper} F \mid X_{F_{v}} \quad \text { is anisotropic }\right\} \\
& S_{i}=\left\{v \in \operatorname{sper} F \mid X_{F_{v}} \quad \text { is isotropic. }\right\}
\end{aligned}
$$

Here $F_{v}$ denotes a real closure of $F$ at $v$.

For $v \in S_{a}, F_{v}(X)$ is not formally real, hence $I^{m} F_{v}(X)=0$. For $v \in S_{i}$, the extension $F_{v}(X) / F_{v}$ is purely transcendental, hence the map

$$
I^{m} F_{v} \rightarrow I_{\mathrm{nr}}^{m}\left(F_{v}(X) / F_{v}\right)
$$

is an isomorphism (compare [17, Prop. 2.5]).

3.6. Lemma. Under the above identification, the image of the map

$$
\theta: I_{\mathrm{nr}}^{m}(F(X) / F) \longrightarrow \prod_{v \in \text { sper } F} I_{\mathrm{nr}}^{m}\left(F_{v}(X) / F_{v}\right) \simeq \prod_{v \in S_{i}} I^{m} F_{v} \stackrel{\left(s g n_{v}\right)}{\longrightarrow}\left(2^{m} \mathbf{Z}\right)^{S_{i}}
$$

is contained in $C\left(S_{i}, 2^{m} \mathbf{Z}\right)$.

Proof. The projection map

$$
\pi: \operatorname{sper} F(X) \rightarrow \operatorname{sper} F
$$


has image $S_{i}$. Let $q \in I_{\mathrm{nr}}^{m}(F(X) / F)$. It is clear that, for any $v \in \operatorname{sper} F(X)$, $\operatorname{sgn}_{v}(q)=\theta(q)(\pi(v))$. In other terms, the following diagram commutes:

$$
\begin{aligned}
& \operatorname{sperF}(X) \stackrel{\pi}{\longrightarrow} S_{i} \\
& s_{q} \searrow \theta(q) \downarrow \\
& 2^{m} \mathbf{Z}
\end{aligned}
$$

where $s_{q}$ is the map $v \mapsto s g n_{v}(q)$. Since $s_{q}$ is continuous, $\operatorname{sper} F(X)$ compact and $\pi$ surjective, it follows that $\theta(q)$ is continuous too.

End of proof of Theorem 4. Let $\left.q \in I_{\mathrm{nr}}^{m} F(X) / F\right)$ and $\bar{s}_{q} \in C\left(S_{i}, 2^{m} \mathbf{Z}\right)$ be the element associated to it by Lemma 3.6. Extend $s_{q}$ as an element of $C\left(\operatorname{sper} F, 2^{m} \mathbf{Z}\right.$ ) (still denoted by $s_{q}$ ) by mapping $S_{a}$ to 0 . As noted above this defines a (unique) element $q_{0} \in I^{m} F$. The images of $\left(q_{0}\right)_{F(X)}$ and $q$ in $C\left(\operatorname{sper} F(X), 2^{m} \mathbf{Z}\right)$ coincide by construction; since $I^{m} F(X) \rightarrow$ $C\left(\operatorname{sper} F(X), 2^{m} \mathbf{Z}\right)$ is injective, we have $q=\left(q_{0}\right)_{F(X)}$ as desired.

\section{Real quadrics}

In this section, we specialise to the case when the ground field is $\mathbf{R}$. We denote by $Q_{d}$ the real anisotropic quadric of dimension $d$, defined by the form $(d+2) .<1>$. Recall that the quadrics can be grouped according to the level of their function fields, and all quadrics in a group are Pfister neighbours of the maximal dimensional quadric in that group, which is defined by a Pfister form $[6, \S 3]$. Further, the level of the function fields in the group $B_{i}$ is $2^{i-1}$, and $H_{n r}^{k}\left(\mathbf{R}\left(Q_{d}\right)\right)=0$ for all $k \geq i$, and all the quadrics $Q_{d}$ in $B_{i}$. We shall freely use the fact that by the results of the previous section, the natural map $H^{n}(\mathbf{R}, \mathbf{Z} / 2) \rightarrow H_{n r}^{n}\left(\mathbf{R}\left(Q_{d}\right), \mathbf{Z} / 2\right)$ is surjective for all $n$. We shall also use without mention, the results from [17, Prop. 1.1] on the Chow groups of quadrics. Recall also that ${ }_{2} \mathrm{CH}^{3}\left(Q_{d}\right) \simeq \mathbf{Z} / 2$ if and only if $Q_{d}$ is defined by a 3-Pfister neighbour [19, Th. 6.1]. We denote the étale cohomology groups $H_{e t}^{n}(X, \mathbf{Z} / 2)$ of a variety $X$ by $H^{n}(X)$. Finally, we shall view the quadric $Q_{d-1}$ as a hyperplane section in $Q_{d}$ and denote the affine open complement by $U_{d}$. We first compute the étale cohomology groups of the real quadrics.

4.1. Lemma. The natural map $H^{n}(\mathbf{R}) \rightarrow H^{n}\left(U_{d}\right)$ is an isomorphism for $0 \leq n \leq d$, and $H^{n}\left(U_{d}\right)=0$ for $n>d$.

Proof. Let $\bar{U}_{d}$ be the complex quadric $U_{d} \times_{\mathbf{R}} \mathbf{C}$. It is well-known [8] that $H^{i}\left(\bar{U}_{d}\right)=0$ for $i \neq 0, d$, and $H^{0}\left(\bar{U}_{d}\right) \simeq H^{d}\left(\bar{U}_{d}\right) \simeq \mathbf{Z} / 2$. On the other 
hand, by Cox's Theorem [7, Th. 2.1], $H^{i}\left(U_{d}\right)=0$ for $i$ large, because $U_{d}$ has no real points. Using this in the long exact sequence

$$
\cdots \longrightarrow H^{i}\left(U_{d}\right) \longrightarrow H^{i}\left(\bar{U}_{d}\right) \longrightarrow H^{i}\left(U_{d}\right) \stackrel{\cup(-1)}{\longrightarrow} H^{i+1}\left(U_{d}\right) \longrightarrow \ldots
$$

we see that the lemma follows, since the natural map $H^{0}(\mathbf{R}) \rightarrow H^{0}\left(U_{d}\right)$ is an isomorphism.

4.2. Lemma. Let $X$ be a smooth projective variety of pure dimension $d$ over $\mathbf{R}$, with no real points. Then the trace map induces an isomorphism

$$
H^{2 d}(X) \stackrel{\sim}{\rightarrow} \mathbf{Z} / 2
$$

and, via this isomorphism, cup-product induces perfect pairings

$$
H^{i}(X) \times H^{2 d-i}(X) \rightarrow \mathbf{Z} / 2 .
$$

Proof. (We thank O. Gabber for pointing out this proof) By [7, Prop. 1.2], there are natural isomorphisms

$$
H_{\mathrm{et}}^{i}(X) \simeq H^{i}\left(X(\mathbf{C}) \times_{\mathbf{Z} / 2} E \mathbf{Z} / 2\right)
$$

where the right hand side is singular cohomology, $\mathbf{Z} / 2$ acts on $X(\mathbf{C})$ via $\operatorname{Gal}(\mathbf{C} / \mathbf{R})$ and $E \mathbf{Z} / 2$ is some contractible space on which $\mathbf{Z} / 2$ acts freely. Since $X(\mathbf{R})=\emptyset$, the action of $\mathbf{Z} / 2$ on $X(\mathbf{C})$ is free and the first projection induces a homotopy equivalence

$$
X(\mathbf{C}) \times_{\mathbf{Z} / 2} E \mathbf{Z} / 2 \approx Y
$$

with $Y:=X(\mathbf{C}) /(\mathbf{Z} / 2)$. Then $Y$ is a smooth compact real manifold of dimension $2 d$, and the result follows from standard Poincaré duality.

The following proposition was announced in [17].

4.3. Proposition. We have

$$
\operatorname{dim} H^{n}\left(Q_{d}\right)= \begin{cases}{\left[\frac{n}{2}\right]+1} & \text { if } d \geq n \\ {\left[\frac{2 d-n}{2}\right]+1} & \text { if } d \leq n .\end{cases}
$$


Proof. We use the Gysin exact sequence

$$
\rightarrow H^{i-2}\left(Q_{d-1}\right) \rightarrow H^{i}\left(Q_{d}\right) \rightarrow H^{i}\left(U_{d}\right) \rightarrow H^{i-1}\left(Q_{d-1}\right) \rightarrow \ldots
$$

for $Q_{d-1} \hookrightarrow Q_{d}$ and the affine open complement $U_{d}$. By Lemma 4.1, the restriction map $H^{i}\left(Q_{d}\right) \rightarrow H^{i}\left(U_{d}\right)$ is surjective for $i \leq d$. Therefore we get short exact sequences

$$
0 \rightarrow H^{i-2}\left(Q_{d-1}\right) \rightarrow H^{i}\left(Q_{d}\right) \rightarrow H^{i}\left(U_{d}\right) \rightarrow 0 \text { for } 2 \leq i \leq d,
$$

and $H^{i}\left(Q_{d}\right) \simeq H^{i-2}\left(Q_{d-1}\right)$ for $d<i \leq 2 d$. Further, by Lemma 4.2 we have $\operatorname{dim} H^{n}\left(Q_{d}\right)=\operatorname{dim} H^{2 d-n}\left(Q_{d}\right)$. An induction argument now completes the proof.

Recall the Bloch-Ogus spectral sequence

$$
E_{2}^{p, q}=H^{p}\left(X, \mathcal{H}_{q}\right) \Longrightarrow H^{n}(X)
$$

for $X$ a smooth variety over $k$. We now compute some $E_{2}^{p, q}$ terms of the Bloch-Ogus spectral sequence for $Q_{d}$. Let us first record the results of [17, Appendix B]:

4.4. Theorem. a) For any $d \geq 1$, we have:

$$
\operatorname{dim} H^{1}\left(Q_{d}, \mathcal{H}^{2}\right)+\operatorname{dim} H^{0}\left(Q_{d}, \mathcal{H}^{3}\right)=\operatorname{dim} H^{3}\left(Q_{d}\right) ;
$$

$\operatorname{dim} C H^{2} Q_{d} \otimes \mathbf{Z} / 2+\operatorname{dim} H^{1}\left(Q_{d}, \mathcal{H}^{3}\right)+\operatorname{dim} H^{0}\left(Q_{d}, \mathcal{H}^{4}\right)=\operatorname{dim} H^{4}\left(Q_{d}\right)$.

b) The "edge homomorphism" $H^{2}\left(Q_{d}, \mathcal{H}^{3}\right) \rightarrow H^{5}\left(Q_{d}\right)$ is injective.

c) We have:

$$
\operatorname{dim} H^{1}\left(Q_{d}, \mathcal{H}^{2}\right)= \begin{cases}0 & \text { for } d=1 \\ 1 & \text { for } d=2 \\ 2 & \text { for } 3 \leq d \leq 6 \\ 1 & \text { for } d \geq 7\end{cases}
$$

and

$$
\operatorname{dim} H^{1}\left(Q_{d}, \mathcal{H}^{3}\right)= \begin{cases}0 & \text { for } d \leq 3 \\ 1 & \text { for } 4 \leq d \leq 6 \\ 2 & \text { for } 7 \leq d \leq 14 \\ 1 & \text { for } d \geq 15\end{cases}
$$

Moreover, Theorem 3 implies the following generalisation of [17, Th. B.1] (using the results on level recalled above and noting that $H_{n r}^{n}\left(\mathbf{R}\left(Q_{d}\right)\right)=$ $H^{0}\left(Q_{d}, \mathcal{H}^{n}\right)[4]$ : 
4.5. Theorem. For any $i, d>0$, the map $H^{i}(\mathbf{R}) \rightarrow H^{0}\left(Q_{d}, \mathcal{H}^{i}\right)$ is surjective. If $d>2^{i}-2$, it is bijective; if $d \leq 2^{i}-2, H^{0}\left(Q_{d}, \mathcal{H}^{i}\right)=0$.

We shall now prove the following result which is a higher analogue of Theorem 4.4:

4.6. Theorem. We have

a)

$$
\operatorname{dim} H^{2}\left(Q_{d}, \mathcal{H}^{3}\right)= \begin{cases}0 & \text { for } d \leq 2 \\ 1 & \text { for } d=3 \\ 2 & \text { for } d=4 \\ 3 & \text { for } 5 \leq d \leq 7 \\ 2 & \text { for } 8 \leq d \leq 14 \\ 1 & \text { for } d \geq 15\end{cases}
$$

and

$$
\operatorname{dim} H^{1}\left(Q_{d}, \mathcal{H}^{4}\right)= \begin{cases}0 & \text { for } d \leq 7 \\ 1 & \text { for } 8 \leq d \leq 14 \\ 2 & \text { for } 15 \leq d \leq 30 \\ 1 & \text { for } d \geq 31\end{cases}
$$

b) The cycle map $\mathrm{cl}^{3}: \mathrm{CH}^{3}\left(Q_{d}\right) / 2 \rightarrow H^{6}\left(Q_{d}\right)$ is injective for all $d$.

c) $\operatorname{dim} H^{1}\left(Q_{d}, \mathcal{H}^{4}\right)+\operatorname{dim} H^{2}\left(Q_{d}, \mathcal{H}^{3}\right)+\operatorname{dim} H^{0}\left(Q_{d}, \mathcal{H}^{5}\right)=\operatorname{dim} H^{5}\left(Q_{d}\right)$.

d) The "edge homomorphism" $H^{2}\left(Q_{d}, \mathcal{H}^{4}\right) \rightarrow H^{5}\left(Q_{d}\right)$ is injective for all $d$.

Proof. We shall repeatedly use the long exact localisation sequence

$$
\begin{aligned}
0 \rightarrow H^{0}\left(Q_{d}, \mathcal{H}^{i}\right) & \rightarrow H^{0}\left(U_{d}, \mathcal{H}^{i}\right) \rightarrow H^{0}\left(Q_{d-1}, \mathcal{H}^{i-1}\right) \\
& \rightarrow H^{1}\left(Q_{d}, \mathcal{H}^{i}\right) \rightarrow H^{1}\left(U_{d}, \mathcal{H}^{i}\right) \rightarrow \cdots .
\end{aligned}
$$

Recall that for all $d$, the map $H^{2}\left(Q_{d}, \mathcal{H}^{3}\right) \rightarrow H^{5}\left(Q_{d}\right)$ of the Bloch-Ogus spectral sequence is injective ( $c f$. Th. $4.4 \mathrm{~b}$ )). Our basic strategy will be to analyse when this map is an isomorphism, and we will do this by computing the dimension of $H^{2}\left(Q_{d}, \mathcal{H}^{3}\right)$ in each case. Note that by the results on $H^{0}\left(Q_{d}, \mathcal{H}^{5}\right)$, the contributions to $H^{5}\left(Q_{d}\right)$ (resp. $\left.H^{5}\left(U_{d}\right)\right)$ in the BlochOgus spectral sequence can be from $E_{\infty}^{2,3}\left(Q_{d}\right)$ and $E_{\infty}^{1,4}\left(Q_{d}\right)\left(\right.$ resp. $E_{\infty}^{2,3}\left(U_{d}\right)$ and $\left.E_{\infty}^{1,4}\left(U_{d}\right)\right)$. However as $H^{5}\left(U_{d}\right) \simeq \mathbf{Z} / 2$, at least one of these $E_{\infty}$ terms for $U_{d}$ is zero, and the common thread in all the cases is to pin down the 
precise contributing terms for $Q_{d}$ by analysing the spectral sequence for $U_{d}$ and using the localisation sequence (6).

We start with the first non-trivial case $d=3$. In this case, $H^{5}\left(Q_{3}\right) \simeq \mathbf{Z} / 2$ and ${ }_{2} C H^{3}\left(Q_{3}\right)=0$ and $H^{0}\left(Q_{3}, \mathcal{H}^{5}\right)=0$. As $H^{1}\left(U_{3}, \mathcal{H}^{3}\right) \hookrightarrow H^{4}\left(U_{3}\right)$ and the latter group is trivial [Lemma 4.1], using (6) we get an injective map $H^{1}\left(Q_{2}, \mathcal{H}^{2}\right) \hookrightarrow H^{2}\left(Q_{3}, \mathcal{H}^{3}\right)$. But $H^{1}\left(Q_{2}, \mathcal{H}^{2}\right) \simeq \mathbf{Z} / 2$ [Th. 4.4 c)] and hence $H^{2}\left(Q_{3}, \mathcal{H}^{3}\right) \simeq H^{5}\left(Q_{3}\right) \simeq \mathbf{Z} / 2$. Clearly, $c l^{3}$ is injective, $E_{2}^{1,4}\left(Q_{3}\right)=$ $H^{1}\left(Q_{3}, \mathcal{H}^{4}\right)=E_{\infty}^{1,4}\left(Q_{3}\right)$ is trivial.

For $d=4$, we have $\operatorname{dim} H^{5}\left(Q_{4}\right) \simeq 2$ and ${ }_{2} C H^{3}\left(Q_{4}\right) \simeq \mathbf{Z} / 2[19$, Th. 5.5 II]. We claim that $H^{2}\left(Q_{4}, \mathcal{H}^{3}\right)$ has dimension at least 2. To see this, consider the commutative diagram

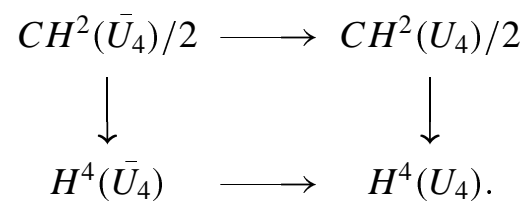

The lower horizontal map is surjective ( $c f$. proof of Lemma 4.1) and the right vertical map is an isomorphism. To prove the latter assertion, we consider the quadrics $C_{d}=Q_{d} \times_{\mathbf{C}} \mathbf{R}$. We have the following commutative diagram of localisation exact sequences

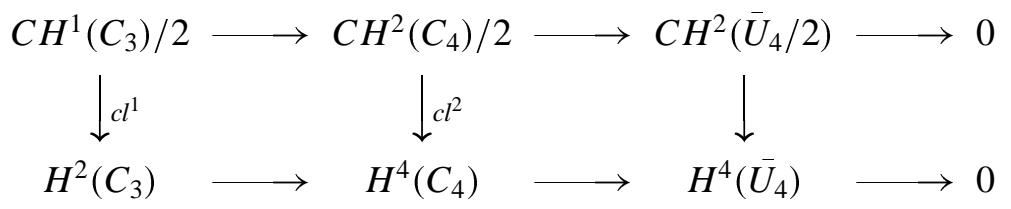

where the exactness of the bottom horizontal row follows from [6, Prop. 2.2]. The cycle maps $c l^{1}$ and $c l^{2}$ are isomorphisms (cf. [17, Prop. 1.5]), hence the right vertical map is also an isomorphism. As all groups in (7) are isomorphic to $\mathbf{Z} / 2$, we see that all maps in this diagram are isomorphisms. This implies that $H^{1}\left(U_{4}, \mathcal{H}^{3}\right)=E_{\infty}^{1,3}\left(U_{4}\right)$ is trivial. The localisation sequence (6) with $d=4, i=3$ therefore gives an inclusion $H^{1}\left(Q_{3}, \mathcal{H}^{2}\right) \hookrightarrow H^{2}\left(Q_{4}, \mathcal{H}^{3}\right)$. Hence our claim is proved and as $H^{2}\left(Q_{4}, \mathcal{H}^{3}\right) \hookrightarrow H^{5}\left(Q_{4}\right)$, these groups are isomorphic. The sequence (6) with $d=4, i=4$ also gives $H^{1}\left(Q_{4}, \mathcal{H}^{4}\right)=0$, and therefore the injectivity of $\mathrm{Cl}^{3}$.

We now treat the case $d=5$, where we have $\operatorname{dim} H^{3}\left(Q_{5}\right)=3$ and ${ }_{2} \mathrm{CH}^{3}\left(Q_{5}\right) \simeq \mathbf{Z} / 2[19, \S 8]$. We shall need the exact sequence $[3,3.6]$

$$
0 \rightarrow H^{2}\left(Q_{d}, \mathcal{K}_{3}\right) / 2 \rightarrow H^{2}\left(Q_{d}, \mathcal{H}^{3}\right) \rightarrow{ }_{2} C H^{3}\left(Q_{d}\right) \rightarrow 0
$$

and the following result of Rost for $d \geq 3$ [25]:

$$
\operatorname{Ker}\left(H^{2}\left(Q_{d}, \mathcal{K}_{3}\right) \rightarrow H^{2}\left(C_{d}, \mathcal{K}_{3}\right)\right) \simeq \operatorname{Ker}\left(H^{4}(\mathbf{R}) \rightarrow H^{4}\left(\mathbf{R}\left(Q_{d}\right)\right)\right) .
$$


In this case, we have a split exact sequence

$0 \rightarrow\left(\operatorname{Ker}\left(H^{4}(\mathbf{R}) \rightarrow H^{4}\left(\mathbf{R}\left(Q_{5}\right)\right)\right) \rightarrow H^{2}\left(Q_{5}, \mathcal{K}_{3}\right) \rightarrow H^{2}\left(C_{5}, \mathcal{K}_{3}\right)^{G} \rightarrow 0\right.$.

Indeed, since $H^{2}\left(C_{5}, \mathcal{K}_{3}\right) \simeq K_{1}(\mathbf{C}) \otimes H^{2}\left(C_{5}, \mathcal{K}_{2}\right)$, hence the last term in the above exact sequence is isomorphic to $\mathbf{R}^{*}$, and the splitting is given by tensoring with the class of the hyperplane section ( $c f$. [17, Prop. 1.1]). Using this in (9), we have $\operatorname{dim} H^{2}\left(Q_{5}, \mathcal{H}^{3}\right)=3$ and hence $H^{2}\left(Q_{5}, \mathcal{H}^{3}\right) \simeq$ $H^{5}\left(Q_{5}\right)$. To see that the cycle map is injective, we use the commutative diagram

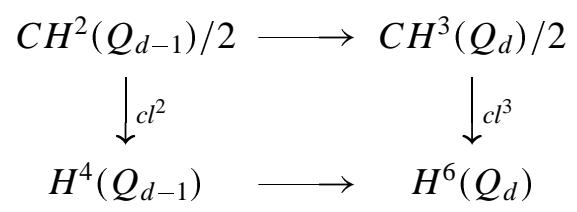

with $d=5$. The left vertical map is injective for all $d$ by [6, Cor. 2.8]. The proof of Prop. 4.3 shows that bottom horizontal map is also injective. In particular the top horizontal map is injective for all $d$. For $d=5$, it is in fact an isomorphism as both sides have the same dimension, and the injectivity of $c l^{3}$ is now clear. Finally, it also emerges from all this discussion that $E_{2}^{1,4}\left(Q_{5}\right)=E_{\infty}^{1,4}\left(Q_{5}\right)=0$.

When $d=6$, for the same reasons as for $Q_{5}$, we have $H^{2}\left(Q_{6}, \mathcal{H}^{3}\right) \simeq$ $H^{5}\left(Q_{6}\right)$. We argue as in the case of $Q_{4}$, but with $C H^{2}$ and $C H^{3}$ in the diagram (8). From the description of the Chow groups of $C_{5}$ and $C_{6}$ (resp. $Q_{5}$ and $\left.Q_{6}\right)[17, \mathrm{I}]$, and the localisation sequence we see that $C H^{3}\left(\bar{U}_{6}\right) / 2 \simeq$ $H^{6}\left(\bar{U}_{6}\right) \simeq \mathbf{Z} / 2$, $\left(\right.$ resp. $\left.C H^{3}\left(U_{6}\right) / 2 \simeq H^{6}\left(U_{6}\right) \simeq \mathbf{Z} / 2\right)$. We use this in the commutative diagram of localisation exact sequences ( $c f$. proof of 4.3)

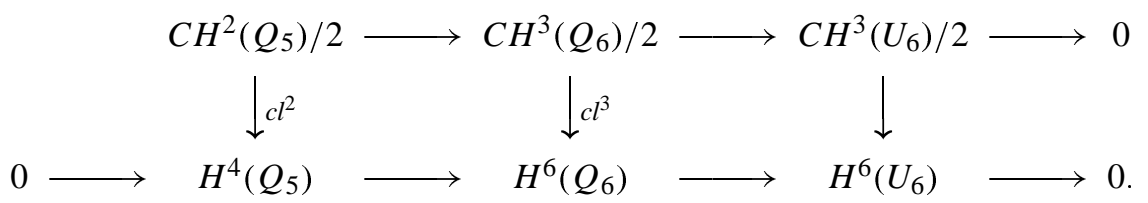

Since $c l^{2}$ is injective, it is plain that $c l^{3}$ is injective. In particular, $E_{2}^{1,4}\left(Q_{6}\right)=$ $E_{\infty}^{1,4}\left(Q_{6}\right)=0$.

For $d=7, \mathrm{CH}^{3}\left(Q_{7}\right) \simeq \mathbf{Z} \cdot h^{3} \oplus \mathbf{Z} / 2$; the existence of torsion can be seen by analysing the invariant $s$ and $G^{*}\left(K\left(Q_{7}\right)\right)$ (cf. [19]). As before, we have $H^{2}\left(Q_{7}, \mathcal{H}^{3}\right) \simeq H^{5}\left(Q_{7}\right) \simeq \mathbf{Z} / 2 \oplus \mathbf{Z} / 2 \oplus \mathbf{Z} / 2$, since the level of $\mathbf{R}\left(Q_{7}\right)$ is 8 . The argument using the commutative diagram (11) gives the injectivity of the cycle map and the vanishing of $H^{1}\left(Q_{7}, \mathcal{H}^{4}\right)$.

In the range $8 \leq d \leq 14$, we have ${ }_{2} C H^{3}\left(Q_{d}\right)=0$. For $d>10$, this follows from Karpenko's result [21], for the remaining ones, it follows from Theorem 1(b), noting that Coker $\eta^{4}$ is trivial for $Q_{d}$, when $8 \leq d \leq 10$, and that the level of the function field is 8 . Thus for all $d$ in the range $8 \leq d \leq 14$, 
we see that $H^{2}\left(Q_{d}, \mathcal{H}^{3}\right)$ has dimension 2. Since $C H^{3}\left(Q_{d}\right) \simeq \mathbf{Z} \cdot h^{3}$, the injectivity of the cycle map is clear. As $H^{5}\left(Q_{d}\right)$ has dimension 3 , we must therefore have $H^{1}\left(Q_{d}, \mathcal{H}^{4}\right)=E_{\infty}^{1,4}\left(Q_{d}\right) \simeq \mathbf{Z} / 2$.

For $d \geq 15$, we have $C H^{3}\left(Q_{d}\right) \simeq \mathbf{Z} \cdot h^{3}$ and that the natural map $H^{4}(R) \rightarrow H^{4}\left(\mathbf{R}\left(Q_{d}\right)\right)$ is injective. Therefore, we see from (9) and (10) that $H^{2}\left(Q_{d}, \mathcal{H}^{3}\right) \simeq \mathbf{Z} / 2$. The injectivity of the cycle map is obvious, hence $H^{1}\left(Q_{d}, \mathcal{H}^{4}\right)=E_{\infty}^{1,4}\left(Q_{d}\right)$. For $15 \leq d \leq 30$, the level of $\mathbf{R}\left(Q_{d}\right)$ is 16 , hence $H_{n r}^{5}\left(Q_{d}\right)=0$, which implies that $H^{1}\left(Q_{d}, \mathcal{H}^{4}\right)$ necessarily has dimension 2. For $d>30$, the map $H^{5}(\mathbf{R}) \rightarrow H^{5}\left(\mathbf{R}\left(Q_{d}\right)\right)$ is injective, hence $H_{n r}^{5}\left(R\left(Q_{d}\right)\right) \simeq H^{5}(\mathbf{R})$, and this implies that $H^{1}\left(Q_{d}, \mathcal{H}^{4}\right) \simeq \mathbf{Z} / 2$.

This completes the proof of a) and b). Assertion c) is obvious, since the above discussion shows that the relevant differentials in the Bloch-Ogus spectral sequence vanish. Assertion d) follows from Theorem 4.5. This completes the proof of the theorem.

4.7. Remark. The computations above actually show that the restriction map $H^{p}\left(Q_{d+1}, \mathcal{H}^{q}\right) \rightarrow H^{p}\left(Q_{d}, \mathcal{H}^{q}\right)$ is an isomorphism for $d$ large, at least for $p+q \leq 5$. This fits with a general conjecture that, for any field $F$ and any quadric $X$ over $F$, the natural map $C H^{p}(X) \otimes H^{q-p}(F) \rightarrow H^{p}\left(X, \mathcal{H}^{q}\right)$ is an isomorphism for $\operatorname{dim} X$ large enough with respect to $p$ and $q$, although we have not checked that this last fact holds for $F=\mathbf{R}$ and $p+q \leq 5$. (This conjecture is true for $p+q \leq 3$ by [14, Th. 1 and 2].)

Finally, let us note the following nice consequence of Theorem 4 and the subform theorem:

Theorem 5. For any $d>0$, the group $W_{\mathrm{nr}}\left(\mathbf{R}\left(Q_{d}\right) / \mathbf{R}\right)$ is cyclic of order $2^{n}$, where $2^{n}$ is the smallest power of 2 which is $\geq d+2$.

Acknowledgements. The results of this paper were found during two stays of the first author in the Tata Institute of Fundamental Research (TIFR) in February-March 1997 and December 1998-February 1999, and in the course of the second author's visit to Universität Regensburg in 1997-98. They were explained in the May 1999 Oberwolfach conference on quadratic forms. The first author wishes to thank TIFR for its usual excellent working conditions while the second author gratefully acknowledges support from the Alexander von Humboldt foundation and the hospitality of Regensburg University.

\section{References}

1. Arason, J.Kr.: Cohomologische Invarianten quadratischer Formen. J. Alg. 36, 448-491 (1975)

2. Arason, J.Kr., Elman, R., Jacob, W.: Graded Witt ring and Galois cohomology, I. CMS Conference Proc. 4, 19-50 (1984), AMS

3. Colliot-Thélène, J.-L.: Cycles algébriques de torsion et $K$-théorie algébrique. Arithmetic algebraic geometry (Trento, 1991), Ballico, E., ed., Lect. Notes Math. 1553, pp. 1-49. Berlin, Heidelberg, New York: Springer 1993 
4. Colliot-Thélène, J.-L.: Birational invariants, purity and the Gersten conjecture. In: Jacob, W., Rosenberg, A. (eds.), $K$-theory and algebraic geometry: connections with quadratic forms and division algebras. Proc. Symp. Pure Math. 58 (I), 1-64 (1995)

5. Colliot-Thélène, J.-L., Ojanguren, M.: Variétés unirationnelles non rationnelles: audelà de l'exemple d'Artin et Mumford. Invent. math. 97, 141-158 (1989)

6. Colliot-Thélène, J.-L., Sujatha, R.: Unramified Witt groups of real anisotropic quadrics. In: Jacob, W., Rosenberg, A. (eds.), $K$-theory and algebraic geometry: connections with quadratic forms and division algebras. Proc. Symp. Pure Math. 58 (II), 127-147 (1995)

7. Cox, D.A.: The étale homotopy type of varieties over R. Proc. AMS 76, 17-22 (1979)

8. Deligne, P.: Quadriques. Groupes de Monodromie en géométrie algébrique (SGA 7), exposé XII. Lect. Notes Math. 340, pp. 62-81, Berlin, Heidelberg, New York: Springer 1973

9. Esnault, H., Kahn, B., Levine, M., Viehweg, E.: The Arason invariant and mod 2 algebraic cycles. J. AMS 11, 73-118 (1998)

10. Hoffmann, D.: Similarity of quadratic forms and half-neighbours. J. Alg. 204, 255-280 (1998)

11. Izhboldin, O.T.: On the non-excellence of function fields extensions. Doc. Math. J. 1, 127-136 (1996)

12. Izhboldin, O.T.: Field with $u$-invariant 9 (preprint 2000), http://www. mathematik.uni-bielefeld.de/LAG/

13. Jacob, W., Rost, M.: Degree four cohomological invariants for quadratic forms. Invent. math. 96, 551-570 (1989)

14. Kahn, B.: Lower $\mathcal{H}$-cohomology of higher-dimensional quadrics. Arch. Math. (Basel) 65, 244-250 (1995)

15. Kahn, B.: A descent problem for quadratic forms. Duke Math. J. 80, 139-155 (1995)

16. Kahn, B.: Motivic cohomology of smooth geometrically cellular varieties. Proc. Symp. Pure Math. 67, 149-174, AMS, Providence 1999

17. Kahn, B., Rost, M., Sujatha, R.: Unramified cohomology of quadrics, I. Amer. J. Math. 120, 841-891 (1998)

18. Kahn, B., Sujatha, R.: Unramified cohomology of quadrics II. To appear in Duke Math. J.

19. Karpenko, N.: Algebro-geometric invariants of quadratic forms (in Russian). Algebrai-Analiz 2, 141-162, 262 (1990). English translation: Leningrad Math. J. 2, 119-138 (1991)

20. Karpenko, N.: Chow groups of quadrics and the stabilization conjecture. Adv. Sov. Math. 4, 3-8 (1991)

21. Karpenko, N.: Chow groups of quadrics and index reduction formula. Nova J. Alg. Geom. 3, 357-379 (1995)

22. Knebusch, M.: Generic splitting of quadratic forms, II. Proc. London Math. Soc. 34, $1-31(1977)$

23. Laghribi, A.: Isotropie de certaines formes quadratiques de dimensions 7 et 8 sur le corps des fonctions d'une quadrique. Duke Math. J. 85, 397-410 (1996)

24. Merkurjev, A.S.: Simple algebras and quadratic forms (in Russian). Izv. Akad. Nauk SSSR 55, 218-224 (1991); English translation: Math. USSR Izv. 38, 215-221 (1992)

25. Merkurjev, A.S.: $K$-theory of simple algebras. In: Jacob, W., Rosenberg, A. (eds.), $K$-theory and algebraic geometry: connections with quadratic forms and division algebras. Proc. Symp. Pure Math. 58 (I), 65-83 (1995)

26. Morel, F.: Suite spectrale d'Adams et invariants cohomologiques des formes quadratiques. C.R. Acad. Sci. Paris 328, 963-968 (1999)

27. Orlov, D., Vishik, A., Voevodsky, V.: Motivic cohomology of Pfister quadrics. (in preparation)

28. Pfister, A.: Quadratische Formen in beliebigen Körpern. Invent. math. 1, 116-132 (1966)

29. Rost, M.: Some new results on Chow groups of quadrics. (preprint 1990) 
30. Scheiderer, C.: Real and étale cohomology. Lect. Notes Math. 1588, Berlin, Heidelberg, New York: Springer 1994

31. Scharlau, W.: Quadratic and Hermitian forms. Grundlehren Math. Wiss., vol. 294, Berlin, Heidelberg, New York: Springer 1985

32. Voevodsky, V.: Triangulated categories of motives over a field. In: Voevodsky, V., Suslin, A., Friedlander, E.M., (eds.), Cycles, Tansfers, and Motivic Homology theories, Annals of Mathematics Studies, Princeton 1999

33. Voevodsky, V.: The Milnor conjecture. (preprint 1996), http://www.math. uiuc.edu/K-theory/

34. Wadsworth, A.R.: Similarity of quadratic forms and isomorphism of their function fields. Trans. Amer. Math. Soc. 208, 352-358 (1975) 